Research Article

\title{
Effectiveness Evaluation of Missile Electromagnetic Launch System Based on ADC Model Improved by EWM-FAHP-ICWGT
}

\author{
Qiaoyang Li $(\mathbb{D})$ and Guiming Chen \\ Xi'an Research Institute of High-Tech, Xi'an 710025, China \\ Correspondence should be addressed to Qiaoyang Li; liqiaoyang2019@163.com
}

Received 12 June 2020; Revised 8 August 2020; Accepted 14 October 2020; Published 29 October 2020

Academic Editor: Pietro Bia

Copyright (c) 2020 Qiaoyang Li and Guiming Chen. This is an open access article distributed under the Creative Commons Attribution License, which permits unrestricted use, distribution, and reproduction in any medium, provided the original work is properly cited.

\begin{abstract}
To solve the key problems of strong infrared radiation, poor continuous combat capability of the system, serious ablation of the launching device, and environmental pollution during missile launch, the electromagnetic launch system (EMLS) has been studied for missile launching tasks. However, most of the current research studies are aimed at specific technical points and lack the overall design and evaluation of the system. Therefore, the designed system may have problems such as excessive cost, lack of economy, or low overall efficiency. In order to complete the overall evaluation of the missile electromagnetic launch system (MEMLS), this article established an ADC model improved by entropy weight method-fuzzy analytic hierarchy process-improved combination weighting model of game theory (EWM-FAHP-ICWGT). The established model integrates subjective and objective weights, eliminating the problem of negative combination coefficients that traditional CWGT may bring, and the average difference in combination weights is the smallest. Difference analysis and differential analysis are also used here to discuss the requirements for the reliability indicator which is mean time between failure (MTBF) and maintainability indicator which is mean time to repair (MTTR) to further improve the effectiveness of the system. Finally, the indicators that have a significant impact on the performance of MEMLS are analyzed, which provides a direction for further research on the system.
\end{abstract}

\section{Introduction}

The electromagnetic launch system (EMLS) is a launching technology that uses electromagnetic energy to convert it into payload kinetic energy [1]. EMLS can convert electrical energy into the kinetic energy required by the load in a short time and push objects to reach a certain speed quickly [2]. EMLS has been widely used in military equipment such as artillery shells, carrier aircraft, and so on due to the advantages of low launch cost, safe handling, strong adaptability, easy energy control, and repeatable rapid launch. However, the application of the electromagnetic launch technology to missiles is still in the demonstration stage and requires a common breakthrough in technology in multiple fields. Missile electromagnetic launch system (MEMLS) refers to changing the traditional cold launching and thermal launching methods, using electromagnetic launching technology to eject missiles from carriers such as launch cylinders, and then using propellants for initial acceleration. MEMLS can effectively solve the key problems of the traditional missile launch system, such as strong infrared radiation which is not conducive to concealment, poor continuous combat capability, serious ablation of the launcher, and environmental pollution.

1.1. Research Novelty and Contribution. At present, most of the research on MEMLS is still the research of core technologies such as pulsed energy storage power supply, pulsed power discharge, motor control, and so on, and the evaluation of the entire system is relatively lacking. When designing a pulse energy storage system for electromagnetic emission, literature [3] studied inductive pulse energy storage systems, literature [4] studied capacitive pulse energy storage systems, and literature [5] studied hybrid pulse energy storage systems. In order to design the best pulse energy storage power supply, literature [6] studied the evaluation system of the discharge effect of pulse power 
discharge supply. The authors have also conducted optimization research on the motor control of the electromagnetic launch system [7], but the existing research focuses most of the energy on the technical research of the details, rather than the optimization of the entire system. Therefore, it is necessary to evaluate the effectiveness of MEMLS to determine the most important part of the system design and provide a theoretical basis for the design of MEMLS to maximize the benefits.

The main contributions of this article are as follows:

(1) The research questions are novel and guide the trend of systematic research. Different from the traditional research on MEMLS from the bottom technical level, the overall effectiveness of the missile electromagnetic launch system is evaluated for the first time, and several indicators that have significant impact on the overall effectiveness of the system are obtained to determine the key points that should be focused on and breakthroughs. At the same time, it is conducive to achieving better system effectiveness with limited research and development (R\&D) funding.

(2) Improving the traditional availability dependability capability (ADC) model. In terms of indicator selection, starting from the MEMLS combat process, indicators are comprehensive and have practical significance. In terms of weight calculation, the entropy weight method-fuzzy analytic hierarchy process (EWM-FAHP) is used to integrate subjective and objective weights, and the improved combination weighting model of game theory (ICWGT) is used to solve the problem that the combination coefficient may be negative. And the average degree of difference is the smallest.

(3) The evaluation model has good scalability and can be further processed. The comprehensive weighting model proposed is suitable for any quantifiable index evaluation problem, and the method of weight combination can be applied to the combination problem of multiple weight sets. In the later stage of the system scheme demonstration, a fitting method can be used to quickly calculate the scheme performance value.

1.2. Research Status. In order to make the demonstrated MEMLS more reasonable and reliable, the effectiveness of the system needs to be evaluated to determine the best demonstration plan. The methods for evaluating the effectiveness of weapon systems mainly include three categories: analytical calculation methods, index evaluation methods, and simulation methods. One of the most versatile and easy to expand and perfect methods is the ADC model. The ADC model is an efficiency analysis model and method proposed by the United States Weapon System Effectiveness Industry Advisory Committee. The system effectiveness evaluated by the ADC model is a measure of the degree to which the system meets the requirements of a specific set of operational functions and is a function of system availability, dependability, and inherent capability.
1.2.1. Improving the ADC Model. In response to the improvement of the ADC model, Shao established the GrayBirth-Death Process ADC (G-BDP-ADC) model using the gray theory and weight distribution model, which solved the problem of evaluating the effectiveness of the LEO satellite communication system [8]. Pirzadeh and Swindlehurst studied the effectiveness of the hybrid ADC model on how to overcome the channel estimation error caused by coarse quantization [9]. Gui established an effectiveness evaluation model of drone driving equipment based on the ADC method [10]. The above literature puts forward some ideas for the improvement of the ADC method, but the method for weighting the indicators at all levels is single, and there is no combination of objective and subjective factors.

1.2.2. Weight Combination Method. In terms of weight combination, Niu et al. determined the combination of the two weighting methods by finding the difference coefficient [11]. Gao et al. obtained the combination weight by using the objective data to modify the weighting method of the subjective data [12]. Zhai et al. achieved the combined weight of the goal through an optimized planning method [13]. The above weight combination model is only suitable for the combination of two weighting methods and has a high dependence on one of the methods, which has certain limitations and cannot guarantee accuracy.

1.3. Description of the Paper Structure. In order to solve the problem of effectiveness evaluation of MEMLS and improve the deficiencies of the above methods, this paper establishes an ADC model improved by EWM-FAHP-ICWGT. The main structure of this paper is as follows. Section 1 introduces the basic concepts and advantages of EMLS and MEMLS and points out the research status and deficiencies of the ADC model and the combined weighting method. Section 2 establishes the effectiveness evaluation model of the system based on the ADC model improved by EWMFAHP-ICWGT; specifically, it describes the ADC model, data normalization processing, EWM, FAHP, and ICWGT. Section 3 uses the established model framework to calculate the basic parameters based on the sample data. Section 4 calculates and discusses the results of the system effectiveness. Section 5 uses difference analysis and differential analysis to study the requirements and effects of further improving system efficiency on reliability and maintainability indicators under the condition of a certain inherent capacity C. Section 6 summarizes the main innovations and conclusions of this paper.

\section{System Effectiveness Evaluation Model}

This section mainly introduces an effectiveness evaluation model for missile electromagnetic launch based on the ADC model improved by EWM-FAHP-ICWGT established in this paper. The steps and methods for establishing the model are shown in Figure 1. 


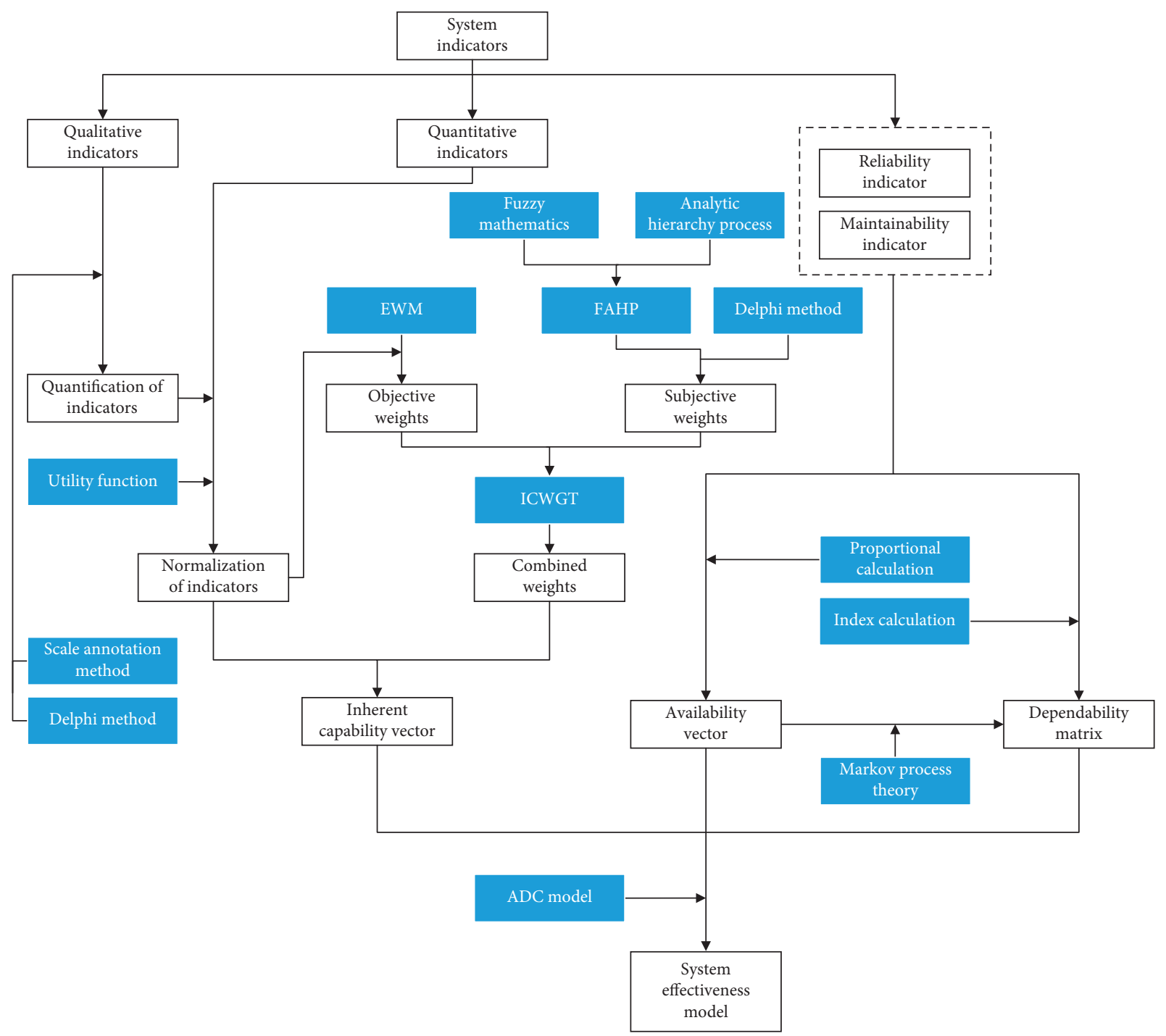

FIGURE 1: Establishment steps and methods of MEMLS effectiveness evaluation model.

2.1. Effectiveness Evaluation Framework Based on ADC Model. First, establish the basic framework of the ADC model, and the general model [14] is

$$
\mathbf{E}=\mathbf{A} \cdot \mathbf{D} \cdot \mathbf{C}
$$

where $\mathbf{E}$ is the effectiveness of the system; $\mathbf{A}$ is the availability vector of the system; D is the dependability matrix of the system; and $\mathbf{C}$ is the inherent capability vector of the system.

Next, the availability vector, dependability matrix, and inherent capability vector model of the system are analyzed and established.

2.1.1. Availability Vector of the System. The availability vector $a$ of the system can be expressed as

$$
\mathbf{A}=\left[a_{1}, a_{2}, a_{3}, \ldots, a_{i}, \ldots, a_{n}\right],
$$

where $a_{i}$ is the probability that the state of the system is $i$ at the initial moment and $n$ is the number of possible states of the system at the initial moment.
The representation of the availability vector model for series and parallel systems $[15,16]$ is discussed below.

(1) Series System. Suppose a system is composed of $m$ subsystems. Each subsystem has only two states: normal and fault. If any of the subsystems fails, the system is faulty. If all subsystems are normal, the system is normal. Such a system is a series system. It is easy to get that the system has only two states: normal and faulty. In this case, the system availability vector is

$$
\begin{aligned}
\mathbf{A}= & {\left[a_{1}, a_{2}\right]=\left[\prod_{i=1}^{m} \frac{\mathrm{MTBF}_{i}}{\mathrm{MTBF}_{i}+\mathrm{MTTR}_{i}+\mathrm{MLDT}_{i}}, 1\right.} \\
& \left.-\prod_{i=1}^{m} \frac{\mathrm{MTBF}_{i}}{\mathrm{MTBF}_{i}+\mathrm{MTTR}_{i}+\mathrm{MLDT}_{i}}\right],
\end{aligned}
$$

where $a_{1}$ is the probability that the initial state of the system is normal; $a_{2}$ is the probability that the initial state of the system is faulty; $\mathrm{MTBF}_{i}$ is the average time between failures 
of the $i$-th subsystem; MTTR $_{i}$ is the average repair time of the $i$-th subsystem; and $\mathrm{MLDT}_{i}$ is the average delay time of the $i$ th subsystem for equipment support. The average delay time of each subsystem is guaranteed.

(2) Parallel System. Suppose a system consists of $m$ subsystems connected in parallel. Each subsystem has only two states: normal and fault. If any of the subsystems is normal, the system is normal. Such a system is a parallel system. It is easy to get that the system has $2^{m}$ states. Note that $a_{\mathrm{si}}$ is the availability of the $i$-th subsystem and $\bar{a}_{\mathrm{si}}=1-a_{\mathrm{si}}$ is the unavailability of the $i$-th subsystem. Then, the availability vector of the system is

$$
\begin{aligned}
\mathbf{A} & =\left[\begin{array}{c}
\left.a_{1}, a_{2}, a_{3}, \ldots, a_{m}, a_{m+1}, a_{m+2}, \ldots, a_{2^{m}}\right] \\
\bar{a}_{s 1} \cdot a_{2} \cdot a_{3} \cdot \ldots \cdot a_{\mathrm{sm}} \\
a_{1} \cdot \bar{a}_{s 2} \cdot a_{3} \cdot \ldots \cdot a_{\mathrm{sm}} \\
\vdots \\
= \\
a_{1} \cdot a_{2} \cdot a_{3} \cdot \ldots \cdot \bar{a}_{\mathrm{sm}} \\
\bar{a}_{s 1} \cdot \bar{a}_{s 2} \cdot a_{3} \cdot \ldots \cdot a_{\mathrm{sm}} \\
\vdots \\
\bar{a}_{s 1} \cdot \bar{a}_{s 2} \cdot \bar{a}_{s 3} \cdot \ldots \cdot \bar{a}_{\mathrm{sm}}
\end{array}\right],
\end{aligned}
$$

where $a_{i}$ is the probability that the system is $i$ at the initial moment; $a_{\mathrm{si}}$ is the availability of the $i$-th subsystem; and $\bar{a}_{\mathrm{si}}=$ $1-a_{\mathrm{si}}$ is the unavailability of the $i$-th subsystem.

2.1.2. Dependability Matrix of the System. Suppose that the number of possible states of the system is $n$; these $n$ states can be converted to each other. According to Markov process theory [17], the state transition matrix is

$$
\mathbf{P}=\left[\begin{array}{cccc}
p_{11} & p_{12} & \cdots & p_{1 n} \\
p_{21} & p_{22} & \cdots & p_{2 n} \\
\vdots & \vdots & \ddots & \vdots \\
p_{n 1} & p_{n 2} & \cdots & p_{n n}
\end{array}\right]
$$

where $\mathbf{P}$ is a transition state matrix and $p_{\mathrm{ij}}$ represents the probability that the state $i$ becomes $j$ after a transition.

If the fault state of the system cannot be repaired temporarily, there is

$$
\mathbf{P}=\left[\begin{array}{cccc}
p_{11} & p_{12} & \ldots & p_{1 n} \\
0 & p_{22} & \ldots & p_{2 n} \\
\vdots & \vdots & \ddots & \vdots \\
0 & 0 & \ldots & 1
\end{array}\right]
$$

Suppose that the number of possible states of the system is $n$; the dependability of the system can be expressed as a state transition matrix:

$$
\mathbf{D}=\left[\begin{array}{cccc}
d_{11}(t) & d_{12}(t) & \ldots & d_{1 n}(t) \\
d_{21}(t) & d_{22}(t) & \ldots & d_{2 n}(t) \\
\vdots & \vdots & \ddots & \vdots \\
d_{n 1}(t) & d_{n 2}(t) & \ldots & d_{\mathrm{nn}}(t)
\end{array}\right]
$$

where $d_{i j}(t)$ represents the probability of transitioning from the $i$ state to the $j$ state at time $t$ when the system is working.

If the system cannot be repaired during the mission, the dependability matrix of the system is expressed as

$$
\mathbf{D}=\left[\begin{array}{cccc}
d_{11}(t) & d_{12}(t) & \ldots & d_{1 n}(t) \\
0 & d_{22}(t) & \ldots & d_{2 n}(t) \\
\vdots & \vdots & \ddots & \vdots \\
0 & 0 & \ldots & 1
\end{array}\right] .
$$

Each element in the matrix meets the following conditions.

$$
\left\{\begin{array}{l}
0 \leq d_{i j}(t) \leq 1, \quad i, j=1,2, \ldots, n, \\
\sum_{j=1}^{n} d_{i j}(t)=1, \quad i=1,2, \ldots, n .
\end{array}\right.
$$

The reliability of series and parallel systems is discussed below. When the system structure is different, the reliability expression of the system is also different. Suppose that $m$ subsystems have independent failures and the reliability of each subsystem is $R_{i}$; then, the reliability of series and parallel systems [18] can be expressed as

$$
\begin{aligned}
& R_{s}(t)=\prod_{i=1}^{m} R_{i}(t), \\
& R_{s}(t)=1-\prod_{i=1}^{m}\left(1-R_{i}(t)\right) .
\end{aligned}
$$

Suppose that the Markov assumption is true; the steps to establish the dependability matrix calculation model are as follows:

(1) Determine the system status.

Combine the different states of the same ability in the process of the system into a state.

(2) Establish a state transition probability matrix $\mathbf{P}$.

Analyze and determine the value of each element in the system state transition matrix.

(3) Establish the system state equation.

The probability that the system is in different states at time $t$ is expressed as

$$
\left\{\begin{array}{c}
p_{1}(t)=\mathrm{P}\left\{\mathrm{S}(t)=\mathrm{S}_{1}\right\} \\
p_{2}(t)=\mathrm{P}\left\{\mathrm{S}(t)=\mathrm{S}_{2}\right\} \\
\vdots \\
p_{n}(t)=\mathrm{P}\left\{\mathrm{S}(t)=\mathrm{S}_{n}\right\}
\end{array}\right.
$$


where $S(t)=S_{\mathrm{i}}$ represents the event that the system state is $i$ at time $t$.

The probability that the system is in a certain state at time $t$ can be expressed by the probability of another state and its state transition probability, that is, after $\Delta t$ time, there is

$$
\mathbf{P}_{j}(t)=p_{i}(t) p_{\mathrm{ij}} \cdot \Delta t
$$

When $\Delta t$ tends to 0 , a set of equations can be obtained.

$$
\left\{\begin{array}{l}
\frac{\mathrm{d} \mathbf{p}(t)}{\mathrm{d} t}=\mathbf{U} \mathbf{p}(t), \\
\mathbf{U}=\left[\mathbf{P}^{T}-\mathbf{I}\right]=\left[\begin{array}{cccc}
p_{11}-1 & p_{12} & \cdots & p_{1 n} \\
p_{21} & p_{22}-1 & \ldots & p_{2 n} \\
\vdots & \vdots & \ddots & \vdots \\
& & & \\
p_{n 1} & p_{n 2} & \ldots & p_{\mathrm{nn}}-1
\end{array}\right], \\
\mathbf{p}(t)=\left[p_{1}(t), p_{2}(t), \ldots, p_{n}(t)\right]^{T} .
\end{array}\right.
$$

(4) Solve the dependability matrix.

The $n$ initial states of the system are also the initial conditions of the system state equation.

$$
\begin{aligned}
\mathbf{p}_{01} & =\left[\begin{array}{c}
p_{1}(0) \\
p_{2}(0) \\
\vdots \\
p_{n}(0)
\end{array}\right]=\left[\begin{array}{c}
1 \\
0 \\
\vdots \\
0
\end{array}\right] ; \mathbf{p}_{02}=\left[\begin{array}{c}
p_{1}(0) \\
p_{2}(0) \\
\vdots \\
p_{n}(0)
\end{array}\right] \\
& =\left[\begin{array}{c}
0 \\
1 \\
\vdots \\
0
\end{array}\right] ; \cdots ; \mathbf{p}_{0 n}=\left[\begin{array}{c}
p_{1}(0) \\
p_{2}(0) \\
\vdots \\
p_{n}(0)
\end{array}\right]=\left[\begin{array}{c}
0 \\
0 \\
\vdots \\
1
\end{array}\right] .
\end{aligned}
$$

From the initial conditions of the system state equation, $n$ special solutions can be obtained.

$$
\mathbf{d}_{1}(t)=\left[\begin{array}{c}
d_{11}(t) \\
d_{12}(t) \\
\vdots \\
d_{1 n}(t)
\end{array}\right] ; \mathbf{d}_{2}(t)=\left[\begin{array}{c}
d_{21}(t) \\
d_{22}(t) \\
\vdots \\
d_{2 n}(t)
\end{array}\right] ; \cdots ; \mathbf{d}_{n}(t)=\left[\begin{array}{c}
d_{n 1}(t) \\
d_{n 2}(t) \\
\vdots \\
d_{\mathrm{nn}}(t)
\end{array}\right] .
$$

From formula (15), the dependability matrix of the system can be obtained as
$\mathbf{D}=\left[\begin{array}{llll}\mathbf{d}_{1}(t) & \mathbf{d}_{2}(t) & \ldots & \mathbf{d}_{n}(t)\end{array}\right]^{T}=\left[\begin{array}{cccc}d_{11}(t) & d_{12}(t) & \ldots & d_{1 n}(t) \\ d_{21}(t) & d_{22}(t) & \ldots & d_{2 n}(t) \\ \vdots & \vdots & \ddots & \vdots \\ d_{n 1}(t) & d_{n 2}(t) & \ldots & d_{\mathrm{nn}}(t)\end{array}\right]$

2.1.3. Inherent Capability Vector of the System. The inherent capability vector $C$ of the system can be expressed as

$$
\mathbf{C}=\left[c_{1}, c_{2}, c_{3}, \ldots, c_{i}, \ldots, c_{n}\right],
$$

where $c_{i}$ is a measure of the ability of the system to complete the task when the state of the system is $i$ during the execution of the task.

If the relevant assumptions hold, then the steps to solve the inherent capability vector of the system are as follows.

(1) Selection of the inherent capability indicators of the system.

The inherent capability indicators of the system play a certain role in completing the task, generally including quantitative and qualitative indicators.. For more complex systems, first-level and second-level indicators can be set up for hierarchical solution.

(2) Quantification of qualitative indicators.

For qualitative indicators, the scale annotation method or Delphi method can be used to score certain performance indicators.

(3) Normalization of indicators after quantization.

The utility function is used to normalize the dimension indicators.

(1) If an index parameter is $x$, the required value is $x_{0}$, and the boundary value is $x^{\prime}$, then there is

If the system effectiveness is positively correlated with $x$, then

$$
R(x)= \begin{cases}0, & x<x^{\prime} \\ \frac{x-x^{\prime}}{x_{0}-x^{\prime}}, & x^{\prime} \leq x<x_{0} \\ 1, & x \geq x_{0} .\end{cases}
$$

If the system effectiveness is negatively correlated with $x$, then

$$
R(x)= \begin{cases}0, & x>x^{\prime} \\ 1-\frac{x-x_{0}}{x^{\prime}-x_{0}}, & x_{0} \leq x<x^{\prime} \\ 1, & x \leq x_{0} .\end{cases}
$$

If $x$ is required to be within a certain range, then $x$ takes a value of 1 within this range, and if it exceeds this range, formulas (18) and (19) are used for processing. 
(2) If the values of different schemes of the same performance indicators are normalized, there are

If the system effectiveness is positively correlated with $x$, then the maximum value is $x^{\prime}$ and

$$
R(x)=\frac{x}{x^{\prime}} .
$$

If the system effectiveness is negatively correlated with $x$, then the minimum value is $x^{\prime}$ and

$$
R(x)=\frac{x^{\prime}}{x}
$$

2.2. Calculating Weights Based on EWM-FAHP. The weight attribute reflects the relative importance of the evaluation indicators, but different weights will lead to different evaluation results; therefore, it is of great value and significance to study the weights of indicators. There are mainly three types of commonly used weighting methods: subjective weighting method, objective weighting method, and combination weighting method. The subjective weighting method is a method for experts in related fields to assign weights to indicators based on their own experience and knowledge. The advantage is that it has greater flexibility, but the disadvantage is that it cannot be explained and there is subjective arbitrariness. The main methods are the Delphi method, analytic hierarchy process (AHP), etc. The objective weighting method refers to the method of weighting based on the relevant mathematical method using the attribute value of the indicator itself. The advantage is that the weight can be interpreted and has a theoretical basis, but the disadvantage is that it lacks certain flexibility and the relevant mathematical model has certain limitations. The main methods are EWM, principal component analysis, gray correlation analysis, rough set theory, etc. Combined weighting refers to a combination method that combines the weights of various methods in order to balance various weighting methods. The main methods are dispersion maximization method, particle swarm optimization method, mathematical programming method, combination weighting model of game theory (CWGT), etc.

In this paper, according to the characteristics of the indicators and the task requirements, we decided to use EWM-FAHP-ICWGT to calculate the combination weight. Its advantage lies in the quantifiable characteristics of second-level indicators. EWM can be used to obtain objective weights, while FAHP can eliminate the lack of basis for consistency testing when calculating subjective weights by AHP. The ICWGT method to calculate the combination weight can solve the problem that the traditional CWGT combination coefficient is negative, and the difference indicator is the smallest compared to other combination methods. On the basis of verifying the reasonableness of the method in this paper, the method of fitting can be used in the later stage of the system design to simplify the calculation process and quickly obtain the performance value of the sample. The steps of the method and the formula used are described in detail below.
2.2.1. Determination of Objective Weights Based on EWM. The concept of entropy was first applied to the field of thermodynamics in physics. EWM originated in 1948. Wiener and Shannon founded information theory. At the same time, the uncertainty of the signal of the information source in the communication process is called information entropy. The proposal of this theory solves the problem of describing information. EWM is a mathematical method for weighting based on the objective information of the indicator. This method calculates the weight based on the comprehensive consideration of the amount of information provided by the indicator. As an objective weighting method, EWM has been widely used in the field of system evaluation.

The steps and methods of calculating the weight of each indicator by information entropy [19] are as follows:

(1) Construct a data matrix.

The dataset composed of $p$ samples and $q$ indicators is normalized to obtain a dataset $X_{i j}$ for evaluation.

$$
\mathbf{X}=\left[\begin{array}{cccc}
x_{11} & x_{12} & \ldots & x_{1 q} \\
x_{21} & x_{22} & \ldots & x_{2 q} \\
\vdots & \vdots & \ddots & \vdots \\
x_{p 1} & x_{p 2} & \ldots & x_{p q}
\end{array}\right]
$$

where $x_{i j}$ is the normalized value of the $j$-th index value in the $i$-th sample.

(2) Calculate the proportion of the $i$-th sample under the $j$-th indicator to all samples of the indicator.

$$
t_{i j}=\frac{x_{i j}}{\sum_{i=1}^{p} x_{i j}}, \quad j=1,2, \ldots, q .
$$

(3) Calculate the entropy of the $j$-th indicator.

$$
e_{j}=\frac{\sum_{i=1}^{p} t_{i j} \ln \left(t_{i j}\right)}{-\ln (p)}, \quad j=1,2, \ldots, q .
$$

(4) Calculate the weight.

The weight is determined by the difference coefficient of the indicator. The greater the difference value of the indicator, the greater the effect on the evaluation and the smaller the entropy value. The weight calculation formula based on EWM can be obtained.

$$
\omega_{1 j}=\frac{1-e_{j}}{\sum_{j=1}^{q}\left(1-e_{j}\right)}, \quad j=1,2, \ldots, q,
$$

where $\omega_{1 j}$ is the EWM weight of the $j$-th index.

2.2.2. Determination of Subjective Weights Based on FAHP. AHP regards the problem of complex multiobjective decision making as a system and decomposes the objective layer into objectives, criteria, and indicators. The AHP uses the expert scoring method to determine the relative importance, and the weight of the indicator is determined by solving the feature vector method [20]. However, the AHP has certain deficiencies, such as the difficulty of consistency testing and 
the lack of a scientific basis for the criterion CR $<0.1$, which is different from the consistency of human thinking. The FAHP introduces the idea of fuzzy mathematics [21] into the traditional AHP. FAHP originated in 1983. Van Loargoven of the Netherlands used the relevant ideas of fuzzy mathematics to fuzzily expand Satty's ranking. Since then, the FAHP has been continuously applied to systematic evaluation and analysis, covering various fields such as risk assessment, analysis of complex environments, industrial production and sales, economic finance, and military technology [22-25]. follows:

The steps to calculate the weights using FAHP are as

(1) Establish a hierarchical analysis structure model.

The establishment of the hierarchical analysis structure model of the system indicators is based on the system identification, and the inherent capability indicators of the system are layered into several groups, and then different levels are formed according to the rules. In the hierarchical structure model, the following hierarchical analysis structure model is mainly established.

(1) Objective layer: indicate the objective of the solution required by AHP.

(2) Criteria layer: generally choose the weight and normalized value of the solution target.

(3) Factor layer: there are many general indicators of the system, so the factor layer often has multiple layers.

(2) Construct fuzzy complementary judgment matrix.

After establishing the hierarchical analysis structure model, the membership relationship between the upper and lower layers is determined accordingly. First, construct a fuzzy judgment matrix. In this paper, the comparison between the two levels of indicators adopts the quantitative expression of the importance of one indicator compared with the other. Generally, the ninescale method in Table 1 is used to get the quantity scale between indexes and establish the fuzzy judgment matrix $\mathbf{F}=\left(f_{i j}\right)_{\mathrm{q} \times \mathrm{q}}$. If $f_{i j}+f_{j i}=1$; then, $F$ is the fuzzy complementary judgment matrix.

(3) Calculate fuzzy consistency judgment matrix and weight vector.

Sum each row of the fuzzy complementary judgment matrix and make a mathematical transformation to obtain the fuzzy consistency judgment matrix $\mathbf{R}=$ $\left(r_{i j}\right)_{\mathrm{q} \times \mathrm{q}}$ as follows:

$$
\left\{\begin{array}{l}
r_{i}=\sum_{j=1}^{q} f_{i j}, \quad i=1,2, \ldots, q, \\
r_{i j}=\frac{r_{i}-r_{j}}{2(q-1)}+\frac{1}{2}, \quad i, j=1,2, \ldots, q .
\end{array}\right.
$$

Perform row and normalization processing on the matrix $\mathbf{R}$ to obtain the indicator ranking vector, in which the weight vector satisfies

$$
\omega_{2 i}=\frac{\sum_{i=1}^{q} f_{i j}-1+(q / 2)}{q(q-1)}, \quad i=1,2, \ldots, q .
$$

(4) Consistency test of fuzzy complementary judgment matrix.

In this paper, when the consistency test is performed, the selection criterion is the compatibility indicator of the fuzzy judgment matrix and its characteristic matrix. Calculate the characteristic matrix of fuzzy judgment matrix.

$$
\left\{\begin{array}{l}
W_{i j}=\frac{\omega_{2 i}}{\omega_{2 i}+\omega_{2 j}}, \quad i, j=1,2, \ldots, q, \\
\mathbf{W}^{*}=\left(W_{i j}\right)_{n \times n}, \quad i, j=1,2, \ldots, q,
\end{array}\right.
$$

where $\mathbf{W}^{*}$ is the characteristic matrix of the fuzzy judgment matrix.

Construct compatibility indicator.

$$
I\left(\mathbf{F}, \mathbf{W}^{*}\right)=\frac{\sum_{i=1}^{q} \sum_{j=1}^{q}\left|f_{i j}-W_{i j}\right|}{q^{2}},
$$

where $I\left(\mathbf{F}, \mathbf{W}^{*}\right)$ is the compatibility indicator.

When formula (30) is satisfied, the judgment matrix is considered to satisfy consistency. The weight obtained by formula (27) is the subjective weight based on FAHP.

$$
I\left(\mathbf{F}, \mathbf{W}^{*}\right) \leq \alpha,
$$

where $\alpha$ represents the attitude of the decision maker. The smaller $\alpha$, the higher the consistency requirement of the decision maker. $\alpha$ is generally 0.1 .

2.3. ICWGT. The weights obtained by EWM are based on the indicator value, which are objective, but unstable, and are greatly affected by data fluctuations. The weights obtained by FAHP reflect the experience of experts, but they are subjectively arbitrary and difficult to guarantee objectivity. How to determine the combined weights under the conditions of taking into account both subjective decision making and objective data is a process of mutual coordination and competition. The object of game theory research is competitive things, and it is a tool for balanced decision making when analyzing the influence of multiple decision-making schemes. The assumption of game theory is that the decision-making schemes are all rational decisions, that is, the decision-making schemes are all positive decisions made to achieve the goals. Game theory seeks to maximize common interests in the decision-making process.

CWGT uses the idea of game theory to synthesize different weighting schemes. Since this game is a non-cooperative game, the basic goal is to minimize the spread between the weighting schemes, that is, to achieve Nash balance.

However, the traditional CWGT is prone to the situation that the obtained combination coefficient is negative, which clearly does not meet the objective requirements. Therefore, this paper uses ICWGT. 
TABLE 1: Nine-scale method.

\begin{tabular}{|c|c|}
\hline Scale value & Value condition \\
\hline 0.5 & Indicator $i$ is as important as indicator $j$ \\
\hline 0.6 & $\begin{array}{c}\text { Indicator } i \text { is slightly more important than } \\
\text { indicator } j\end{array}$ \\
\hline 0.7 & $\begin{array}{c}\text { Indicator } i \text { is obviously more important than } \\
\text { indicator } j\end{array}$ \\
\hline 0.8 & Indicator $i$ is much more important than indicator \\
\hline 0.9 & $\begin{array}{c}\text { Indicator } i \text { is extremely more important than } \\
\text { indicator } j\end{array}$ \\
\hline $\begin{array}{l}0.1,0.2,0.3 \\
0.4\end{array}$ & Inverse comparison of the above indicators \\
\hline
\end{tabular}

Suppose that there are $q$ evaluation indicators and $k$ weighting schemes; $k$ basic weight sets can be obtained, and the linear combination of $k$ basic weight sets can be expressed as

$$
\omega=\sum_{i=1}^{k} \alpha_{i} \omega_{i}^{T}
$$

where $\omega$ is any combination of $k$ basic weight sets; $\alpha_{i}$ represents the linear combination coefficient of the $i$-th weight set, $\alpha_{i}>0$; and $\omega_{i}^{T}$ is the $i$-th weight set.

Based on the basic theory of CWGT [26], the optimized game model is

$$
\min _{j=1,2, \ldots, k}\left\|\sum_{i=1}^{k} \alpha_{i} \omega_{i}^{T}-\omega_{j}\right\|_{2}
$$

where $\omega_{j}$ is the weight set of the $j$-th weighting scheme.

According to the properties of matrix differential, in order to prevent the linear combination coefficient from being negative, the optimal condition of ICWGT is

$$
\min _{\alpha_{1}, \alpha_{2}, \ldots, \alpha_{k}} f=\sum_{j=1}^{k}\left|\left(\sum_{i=1}^{k} \alpha_{i} \omega_{j} \omega_{i}^{T}\right)-\omega_{j} \omega_{j}^{T}\right| .
$$

Borrowing the constraints of the objective weighting method for maximizing dispersion, the linear combination coefficient can also satisfy the following conditions.

$$
\left\{\begin{array}{l}
\alpha_{i}>0, \quad i=1,2, \cdots, k, \\
\sum_{i=1}^{k} \alpha_{i}^{2}=1
\end{array}\right.
$$

The Lagrange function is constructed as follows:

$$
L\left(\alpha_{i}, \lambda\right)=\sum_{j=1}^{k}\left|\left(\sum_{i=1}^{k} \alpha_{i} \omega_{j} \omega_{i}^{T}\right)-\omega_{j} \omega_{j}^{T}\right|+\frac{\lambda}{2}\left(\sum_{i=1}^{k} \alpha_{i}^{2}-1\right) .
$$

Find the partial derivatives of $\alpha_{i}$ and $\lambda$, respectively; combined with the constraints, the partial differential equation can be obtained as follows:

$$
\begin{aligned}
& \frac{\partial L}{\partial \alpha_{i}}=\sum_{j=1}^{k}( \pm) \omega_{j} \omega_{i}^{T}+\lambda \alpha_{i}=0, \\
& \frac{\partial L}{\partial \lambda}=\sum_{i=1}^{k} \alpha_{i}^{2}-1=0 .
\end{aligned}
$$

From formula (36), the following can be obtained:

$$
\alpha_{i}=\frac{\mp \sum_{j=1}^{k} \omega_{j} \omega_{i}^{T}}{\lambda} \text {. }
$$

Substituting formula (38) into formula (37), we can obtain

$$
\lambda= \pm \sqrt{\sum_{i=1}^{k}\left(\sum_{j=1}^{k} \omega_{j} \omega_{i}^{T}\right)^{2}}
$$

Substituting formula (39) into formula (38), when $\alpha_{i}>0$, we can obtain

$$
\alpha_{i}=\frac{\sum_{j=1}^{k} \omega_{j} \omega_{i}^{T}}{\sqrt{\sum_{i=1}^{k}\left(\sum_{j=1}^{k} \omega_{j} \omega_{i}^{T}\right)^{2}}} .
$$

Formula (40) is the only solution that satisfies the condition, and finally $\alpha_{i}$ is normalized as follows:

$$
\alpha_{i}^{*}=\frac{\sum_{j=1}^{k} \omega_{j} \omega_{i}^{T}}{\sum_{i=1}^{k} \sum_{j=1}^{k} \omega_{j} \omega_{i}^{T}} .
$$

The final combination weight is

$$
\omega^{*}=\sum_{i=1}^{k} \alpha_{i}^{*} \omega_{i}^{T}, \quad i=1,2, \ldots, k .
$$

2.4. Rationality Evaluation of Combination Weighting. Different combination methods will get different combination weights, so it is essential to test the rationality of combination weighting. This paper uses the average degree of difference to test the rationality of the combination weights. The formula for calculating the average difference is as follows:

$$
\overline{D\left(\omega^{*}\right)}=\frac{1}{k \times p} \sum_{l=1}^{k} \sum_{i=1}^{p} \sum_{j=1}^{q}\left|x_{\mathrm{ij}} .\left(\omega^{*}(j)-\omega_{l}(j)\right)\right|,
$$

where $x_{\mathrm{ij}}$ is the normalized value of the $j$-th indicator of the $i$ th sample; $\omega^{*}(j)$ is the weight of the $j$-th indicator of the combined weight; $\omega_{l}(j)$ is the weight of the $j$-th indicator of the $l$-th single weighting method; $k$ is the total number of single weighting methods; $p$ is the total number of samples; and $q$ is the total number of indicators.

The smaller the average difference is, the smaller the deviation between the combined weights and the weights obtained by other single weighting methods is, that is, the more reasonable the method of combined weighting. 


\section{Effectiveness Evaluation of MEMLS}

This paper combines the ADC model and AHP to build a hierarchical framework for MEMLS effectiveness evaluation. The overall hierarchy of the evaluation is determined by the AHP method. The target layer is the overall objective of the evaluation, that is, the effectiveness of MEMLS; the criterion layer is determined by the ADC model; the first-level indicator layer is determined by the influence of MEMLS on the missile combat process; the second-level indicator layer is composed of general evaluation indicators of the missile launch system. From the analysis of all elements of the entire process of MEMLS operations, a hierarchy table of MEMLS effectiveness evaluation is obtained, as shown in Table 2.

From Table 2, the target layer is effectiveness evaluation of MEMLS, and the criterion layer is the system availability, dependability, and inherent capability. The inherent ability is divided into 3 first-level indicators and 13 second-level indicators. The effectiveness of the system will be evaluated in accordance with the steps and methods of Figure 1.

3.1. System Availability Analysis. The simple working process of MEMLS is that the energy storage of the pulse energy storage system, the discharge of the pulse power discharge system, and the pulse linear motor work together. Since the working process is a series system and from the perspective of operational decision-making requirements, the division of more states of each subsystem at the initial time will make the system more complicated and not conducive to decision making, so it is assumed that the MEMLS has only two states at the initial time, namely, normal and faulty. According to formula (3), the availability vector of MEMLS is

$$
\begin{gathered}
\mathbf{A}=\left[a_{1}, a_{2}\right]=\left[\frac{\mathrm{MTBF}}{\mathrm{MTBF}+\mathrm{MTTR}+\mathrm{MLDT}}, 1\right. \\
\left.-\frac{\mathrm{MTBF}}{\mathrm{MTBF}+\mathrm{MTTR}+\mathrm{MLDT}}\right],
\end{gathered}
$$

where $a_{1}$ is the probability that the initial state of the system is normal; $a_{2}$ is the probability that the initial state of the system is faulty; MTBF is the average time between failures of the system; MTTR is the average repair time of the system; and MLDT is the average guarantee delay time of the system.

3.2. System Dependability Analysis. Since the working process of MEMLS can be regarded as a Markov process satisfying ergodicity, according to the transition probability property of the Markov process, combined with formulas (14)-(16), the dependability matrix of the system can be obtained as

$$
\mathbf{D}=\left[\begin{array}{cc}
e^{-(t / \mathrm{MTBF})} & 1-e^{-(t / \mathrm{MTBF})} \\
0 & 1
\end{array}\right]
$$

where $t$ is the total duration of the task performed by MEMLS.
3.3. Analysis of Inherent System Capability. At the initial moment, MEMLS only has two states of normal and fault. At the same time, for the battlefield, the ability of the system to complete the task under the fault can be regarded as 0 . Therefore, combining formulas (17) and (44), the inherent capability vector of the system can be obtained as

$$
\mathbf{C}=\left[\begin{array}{l}
c \\
0
\end{array}\right]
$$

where $c$ is the inherent capability value of MEMLS under normal conditions.

In order to calculate $c$, the names and corresponding codes of the first and second indicators of MEMLS selected in this paper are shown in Table 2.

3.3.1. Specific Description and Quantification of Indicators. The following is the description of the secondary indicators of inherent capability.

(1) Dimensional indicators.

(1) Initial ejection velocity $U_{11}$ Initial ejection velocity indicates the speed at which the missile exits the barrel after being accelerated by the launching device. The unit is $\mathrm{m} / \mathrm{s}$.

(2) Acceleration time $U_{12}$

The acceleration time represents the time for the missile to accelerate to a predetermined speed via the guide rail. The unit is ms.

(3) Infrared radiation intensity $U_{21}$ Infrared radiation intensity is an important indicator to measure the missile's stealth performance during the launch process. The unit is W/ sr.

(4) Maintenance and replacement rate of spare parts $U_{33}$

The maintenance and replacement rate of spare parts represents the average time when the spare parts that can be replaced by the launcher are replaced during the execution of the task. The unit is $\mathrm{h}$.

(2) Calculation of quantitative probability indicators. Initial anti-interception rate $U_{23}$ is the probability of anti-interception during the initial phase after the missile is launched by the launch system.

(3) Calculation of qualitative indicators.

The quantification of qualitative indicators is mainly evaluated by the expert scoring method. The scoring range is 0 to 1 . The higher the score, the better. The specific indicators are as follows.

(1) Thrust control accuracy $U_{13}$

Thrust control accuracy indicates how close the thrust of the launching system to the missile is to the required value.

(2) Robustness during launch $U_{14}$ 
TABLE 2: Effectiveness evaluation of MEMLS.

\begin{tabular}{|c|c|c|c|}
\hline Target layer & Criterion layer & $\begin{array}{c}\text { First-level indicator } \\
\text { layer }\end{array}$ & Second-level indicator layer \\
\hline \multirow{9}{*}{$\begin{array}{l}\text { Effectiveness evaluation of } \\
\text { MEMLS }\end{array}$} & $\begin{array}{c}\text { Availability A } \\
\text { Dependability D }\end{array}$ & & \\
\hline & \multirow{8}{*}{ Inherent capability $\mathbf{C}$} & \multirow{6}{*}{ Combat capability $U_{1}$} & $\begin{array}{l}\text { Initial ejection velocity } U_{11} \\
\text { Acceleration time } U_{12} \\
\text { Thrust control accuracy } U_{13}\end{array}$ \\
\hline & & & Robustness during launch $U_{14}$ \\
\hline & & & Continuous combat capability $U_{15}$ \\
\hline & & & Launch system versatility $U_{16}$ \\
\hline & & & Infrared radiation intensity $U_{21}$ \\
\hline & & & Electromagnetic anti-interference ability $U_{22}$ \\
\hline & & Adversarial ability $U_{2}$ & $\begin{array}{l}\text { Initial anti-interception rate } U_{23} \\
\text { Electromagnetic compatibility of own system } U_{24} \\
\text { Ablation degree of launcher } U_{31}\end{array}$ \\
\hline & & State loss $U_{3}$ & $\begin{array}{l}\text { Environmental pollution degree } U_{32} \\
\text { Maintenance and replacement rate of spare parts } \\
\qquad U_{33}\end{array}$ \\
\hline
\end{tabular}

Robustness during launch indicates the degree of stability of the system when the force changes suddenly.

(3) Continuous combat capability $U_{15}$

Continuous combat capability means the ability of the launch system to perform multiple launch missions in a short period of time.

(4) Launch system versatility $U_{16}$ The versatility of the launch system indicates the versatility of the launch system for launching other types of missiles.

(5) Electromagnetic anti-interference ability $U_{22}$ Electromagnetic anti-interference ability represents our antijamming capability in the face of enemy electromagnetic interference during the launch mission.

(6) Electromagnetic compatibility of own system $U_{24}$ Electromagnetic compatibility of the own system indicates the electromagnetic compatibility capability of the transmitter's own system.

(7) Ablation degree of launcher $U_{31}$ The ablation degree of launcher indicates the degree of ablation damage of the launching system to our equipment such as launch vehicle and launch platform.

(8) Environmental pollution degree $U_{32}$

Environmental pollution degree indicates the degree of pollution to the surrounding environment during the mission of the launching system.

3.3.2. Normalization of Quantitative Indicators. The indicator data analyzed in this paper are shown in Table 3. Since the system is being demonstrated, all the data samples used in this paper are derived from the demonstration plan of the research group and the laboratory on the MEMLS and the data of the active missiles using thermal and cold launch systems.
In Table 3, $V_{11}$ to $V_{15}$ are missiles using MEMLS, $V_{21}$ is a missile using a thermal launch system, and $V_{22}$ is a missile using a cold launch system. Combining formulas (20) and (21), the normalized values of the indicators in Table 3 can be obtained, as shown in Table 4.

3.4. Weight Calculation of Inherent Capability. First of all, three first-level indicators are used as subsystems. From Table 4 and formula (22), the evaluation datasets of the three subsystems are

$$
\begin{aligned}
& \mathbf{X}_{1}=\left[\begin{array}{ccccccc}
0.9 & 0.9 & 1 & 1 & 1 & 1 & 0.9 \\
1 & 0.7 & 1 & 1 & 1 & 0.5 & 0.7 \\
0.8 & 0.8 & 0.8 & 0.8 & 0.8 & 0.4 & 0.5 \\
0.8 & 0.8 & 0.8 & 0.8 & 0.8 & 0.5 & 0.6 \\
0.6 & 0.6 & 0.6 & 0.6 & 0.6 & 0.4 & 0.4 \\
0.4 & 0.4 & 0.4 & 0.4 & 0.4 & 0.2 & 0.2
\end{array}\right]^{T}, \\
& \mathbf{X}_{2}=\left[\begin{array}{ccccccc}
1 & 1 & 1 & 1 & 1 & 0.5 & 0.7 \\
0.5 & 0.5 & 0.5 & 0.5 & 0.7 & 0.7 & 0.8 \\
0.8 & 0.8 & 0.8 & 0.8 & 0.8 & 0.7 & 0.7 \\
0.5 & 0.5 & 0.5 & 0.5 & 0.5 & 0.7 & 0.7
\end{array}\right]^{T}, \\
& \mathbf{X}_{3}=\left[\begin{array}{ccccccc}
0.7 & 0.7 & 0.7 & 0.8 & 0.7 & 0.2 & 0.4 \\
0.9 & 0.9 & 0.9 & 0.9 & 0.9 & 0.4 & 0.6 \\
1 & 1 & 1 & 1 & 1 & 0.5 & 0.6
\end{array}\right]^{T},
\end{aligned}
$$

where $\mathbf{X}_{1}$ is the evaluation dataset of combat capability $U_{1}$; $\mathbf{X}_{2}$ is the evaluation dataset of adversarial ability $U_{2}$; and $\mathbf{X}_{3}$ is the evaluation dataset of state loss $U_{3}$.

From formulas (23)-(25), $e_{j}$ and $\omega_{1 j}$ of the indicators based on EWM corresponding to the three subsystems are calculated, respectively, as shown in Table 5. 
TABle 3: Data samples.

\begin{tabular}{lcccccccc}
\hline $\begin{array}{l}\text { First-level } \\
\text { indicators' } \\
\text { code }\end{array}$ & $\begin{array}{c}\text { Second-level } \\
\text { indicators' } \\
\text { code }\end{array}$ & $V_{11}$ & $V_{12}$ & $V_{13}$ & $V_{14}$ & $V_{15}$ & $V_{21}$ & $V_{22}$ \\
\hline & $U_{11}$ & 32 & 32 & 35 & 35 & 35 & 35 & 32 \\
& $U_{12}$ & 10 & 15 & 10 & 10 & 10 & 20 & 15 \\
$U_{1}$ & $U_{13}$ & 0.8 & 0.8 & 0.8 & 0.8 & 0.8 & 0.4 & 0.5 \\
& $U_{14}$ & 0.8 & 0.8 & 0.8 & 0.8 & 0.8 & 0.5 & 0.6 \\
& $U_{15}$ & 0.6 & 0.6 & 0.6 & 0.6 & 0.6 & 0.4 & 0.4 \\
& $U_{16}$ & 0.4 & 0.4 & 0.4 & 0.4 & 0.4 & 0.2 & 0.2 \\
\hline & $U_{21}$ & 10 & 10 & 10 & 10 & 10 & 20 & 15 \\
$U_{2}$ & $U_{22}$ & 0.5 & 0.5 & 0.5 & 0.5 & 0.7 & 0.7 & 0.8 \\
& $U_{23}$ & 0.8 & 0.8 & 0.8 & 0.8 & 0.8 & 0.7 & 0.7 \\
& $U_{24}$ & 0.5 & 0.5 & 0.5 & 0.5 & 0.5 & 0.7 & 0.7 \\
\hline \multirow{3}{*}{$U_{3}$} & $U_{31}$ & 0.7 & 0.7 & 0.7 & 0.8 & 0.7 & 0.2 & 0.4 \\
& $U_{32}$ & 0.9 & 0.9 & 0.9 & 0.9 & 0.9 & 0.4 & 0.6 \\
& $U_{33}$ & 2 & 2 & 2 & 2 & 2 & 4 & 3.5 \\
\hline
\end{tabular}

TABLE 4: Normalized values of data samples.

\begin{tabular}{lcccccccc}
\hline $\begin{array}{l}\text { First-level } \\
\text { indicators } \\
\text { code }\end{array}$ & $\begin{array}{c}\text { Second-level } \\
\text { indicators' } \\
\text { code }\end{array}$ & $V_{11}$ & $V_{12}$ & $V_{13}$ & $V_{14}$ & $V_{15}$ & $V_{21}$ & $V_{22}$ \\
\hline & $U_{11}$ & 0.9 & 0.9 & 1 & 1 & 1 & 1 & 0.9 \\
& $U_{12}$ & 1 & 0.7 & 1 & 1 & 1 & 0.5 & 0.7 \\
$U_{1}$ & $U_{13}$ & 0.8 & 0.8 & 0.8 & 0.8 & 0.8 & 0.4 & 0.5 \\
& $U_{14}$ & 0.8 & 0.8 & 0.8 & 0.8 & 0.8 & 0.5 & 0.6 \\
& $U_{15}$ & 0.6 & 0.6 & 0.6 & 0.6 & 0.6 & 0.4 & 0.4 \\
& $U_{16}$ & 0.4 & 0.4 & 0.4 & 0.4 & 0.4 & 0.2 & 0.2 \\
\hline & $U_{21}$ & 1 & 1 & 1 & 1 & 1 & 0.5 & 0.7 \\
$U_{2}$ & $U_{22}$ & 0.5 & 0.5 & 0.5 & 0.5 & 0.7 & 0.7 & 0.8 \\
& $U_{23}$ & 0.8 & 0.8 & 0.8 & 0.8 & 0.8 & 0.7 & 0.7 \\
& $U_{24}$ & 0.5 & 0.5 & 0.5 & 0.5 & 0.5 & 0.7 & 0.7 \\
\hline \multirow{3}{*}{$U_{3}$} & $U_{31}$ & 0.7 & 0.7 & 0.7 & 0.8 & 0.7 & 0.2 & 0.4 \\
& $U_{32}$ & 0.9 & 0.9 & 0.9 & 0.9 & 0.9 & 0.4 & 0.6 \\
& $U_{33}$ & 1 & 1 & 1 & 1 & 1 & 0.5 & 0.6 \\
\hline
\end{tabular}

From Table 5, the weights of the indicators of each subsystem based on EWM can be obtained, as shown in Figures 2-4.

The weights based on FAHP are calculated below. According to Table 1 and Delphi method, the fuzzy complementary judgment matrices of the three subsystems can be obtained as

$$
\begin{aligned}
B_{1} & =\left[\begin{array}{llllll}
0.5 & 0.6 & 0.7 & 0.7 & 0.8 & 0.8 \\
0.4 & 0.5 & 0.6 & 0.6 & 0.7 & 0.7 \\
0.3 & 0.4 & 0.5 & 0.5 & 0.6 & 0.7 \\
0.3 & 0.4 & 0.5 & 0.5 & 0.6 & 0.7 \\
0.2 & 0.3 & 0.4 & 0.4 & 0.5 & 0.6 \\
0.2 & 0.3 & 0.3 & 0.3 & 0.4 & 0.5
\end{array}\right], \\
B_{2} & =\left[\begin{array}{llll}
0.5 & 0.7 & 0.8 & 0.8 \\
0.3 & 0.5 & 0.6 & 0.6 \\
0.2 & 0.4 & 0.5 & 0.5 \\
0.2 & 0.4 & 0.5 & 0.5
\end{array}\right],
\end{aligned}
$$

TABLE 5: Calculation results based on EWM.

\begin{tabular}{lccc}
\hline $\begin{array}{l}\text { First-level indicators' } \\
\text { code }\end{array}$ & $\begin{array}{c}\text { Second-level indicators' } \\
\text { code }\end{array}$ & $e_{j}$ & $\omega_{1 j}$ \\
\hline & $U_{11}$ & 0.9993 & 0.0108 \\
& $U_{12}$ & 0.9858 & 0.2209 \\
& $U_{13}$ & 0.9851 & 0.2322 \\
$U_{1}$ & $U_{14}$ & 0.9930 & 0.1089 \\
& $U_{15}$ & 0.9924 & 0.1179 \\
& $U_{16}$ & 0.9802 & 0.3093 \\
\hline & $U_{21}$ & 0.9871 & 0.4279 \\
$U_{2}$ & $U_{22}$ & 0.9901 & 0.3289 \\
& $U_{23}$ & 0.9991 & 0.0297 \\
& $U_{24}$ & 0.9935 & 0.2135 \\
\hline \multirow{3}{*}{$U_{3}$} & $U_{31}$ & 0.9658 & 0.5134 \\
& $U_{32}$ & 0.9833 & 0.2511 \\
& $U_{33}$ & 0.9843 & 0.2355 \\
\hline
\end{tabular}

$$
B_{3}=\left[\begin{array}{lll}
0.5 & 0.7 & 0.7 \\
0.3 & 0.5 & 0.6 \\
0.3 & 0.4 & 0.5
\end{array}\right] \text {. }
$$

From formula (26), the fuzzy consistency judgment matrix can be obtained as

$$
\begin{aligned}
R_{1} & =\left[\begin{array}{llllll}
0.5000 & 0.5600 & 0.6100 & 0.6100 & 0.6700 & 0.7100 \\
0.4400 & 0.5000 & 0.5500 & 0.5500 & 0.6100 & 0.6500 \\
0.3900 & 0.4500 & 0.500 & 0.5000 & 0.5600 & 0.6000 \\
0.3900 & 0.4500 & 0.500 & 0.5000 & 0.5600 & 0.6000 \\
0.3300 & 0.3900 & 0.4400 & 0.4400 & 0.5000 & 0.5400 \\
0.2900 & 0.3500 & 0.4000 & 0.4000 & 0.4600 & 0.5000
\end{array}\right], \\
R_{2} & =\left[\begin{array}{llll}
0.5000 & 0.6333 & 0.7000 & 0.7000 \\
0.3667 & 0.5000 & 0.5667 & 0.5667 \\
0.3000 & 0.4333 & 0.5000 & 0.5000 \\
0.3000 & 0.4333 & 0.5000 & 0.5000
\end{array}\right], \\
R_{3} & =\left[\begin{array}{lll}
0.5000 & 0.6250 & 0.6750 \\
0.3750 & 0.5000 & 0.5500 \\
0.3250 & 0.4500 & 0.5000
\end{array}\right] .
\end{aligned}
$$

From formula (27), the weight vectors of the indicators of each subsystem based on FAHP are

$$
\begin{aligned}
& \omega_{21}=(0.2033,0.1833,0.1667,0.1667,0.1467,0.1333), \\
& \omega_{22}=(0.3167,0.2500,0.2167,0.2167), \\
& \omega_{23}=(0.4000,0.3167,0.2833) .
\end{aligned}
$$

The consistency test of the fuzzy complementary judgment matrix is performed as follows, and the characteristic matrix of the fuzzy judgment matrix of each subsystem is calculated by formula (28) as 


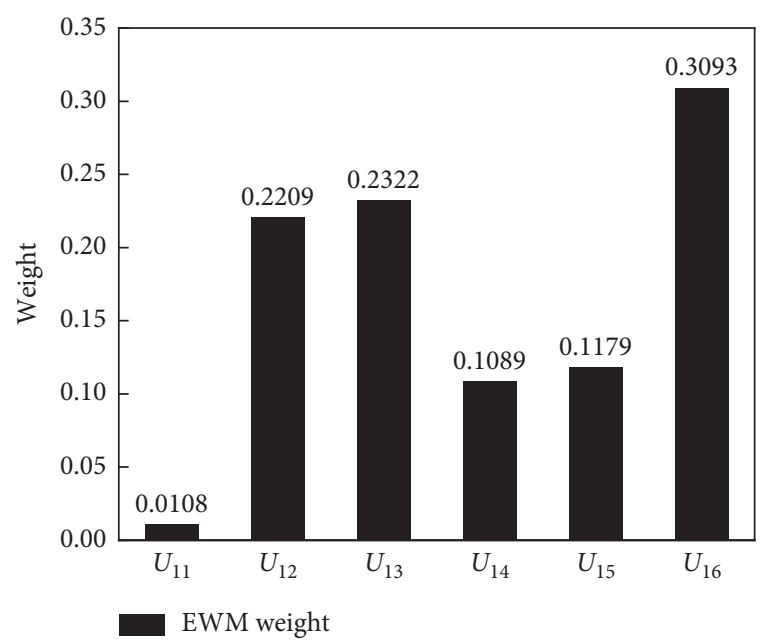

FIGURE 2: Weight of each indicator of combat capability based on EWM.

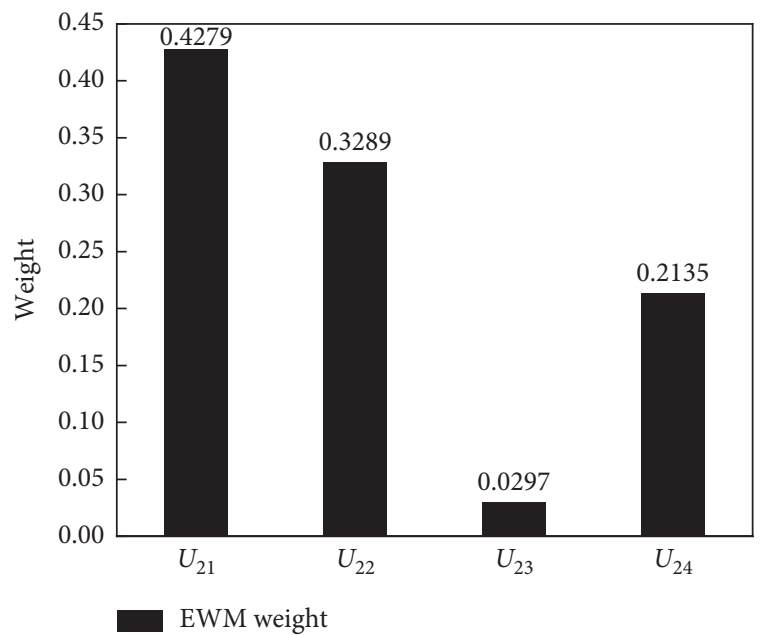

FIGURE 3: Weight of each indicator of adversarial ability based on EWM.

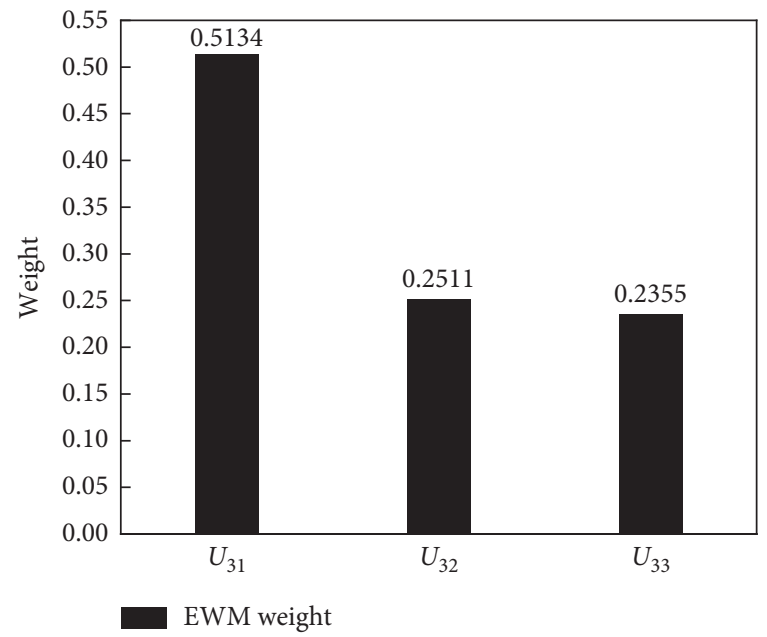

FIgURE 4: Weight of each indicator of state loss based on EWM.

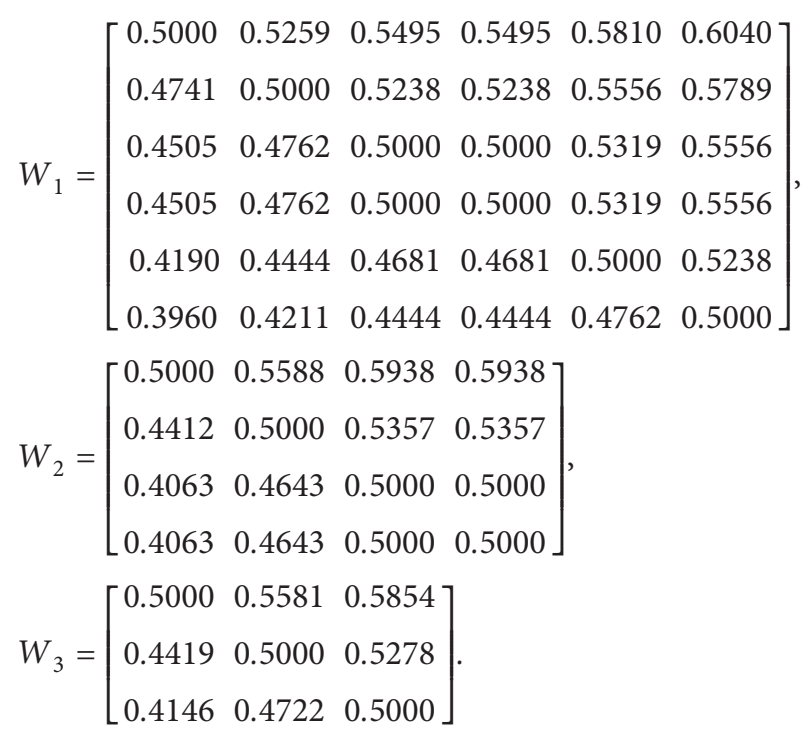

(51)

The compatibility indicators are obtained as

$$
\begin{gathered}
I\left(B_{1}, W_{1}\right)=\frac{1}{n^{2}} \sum_{i=1}^{n} \sum_{j=1}^{n}\left|B_{1 \mathrm{ij}}-W_{1 \mathrm{ij}}\right|=0.0950<0.1, \\
I\left(B_{2}, W_{2}\right)=\frac{1}{n^{2}} \sum_{i=1}^{n} \sum_{j=1}^{n}\left|B_{2 \mathrm{ij}}-W_{2 \mathrm{ij}}\right|=0.0853<0.1, \\
I\left(B_{3}, W_{3}\right)=\frac{1}{n^{2}} \sum_{i=1}^{n} \sum_{j=1}^{n}\left|B_{3 \mathrm{ij}}-W_{3 \mathrm{ij}}\right|=0.0730<0.1 .
\end{gathered}
$$

The inspections are all qualified, and the weights of the indicators of each subsystem based on FAHP are obtained as shown in Figures 5-7.

According to formulas (31)-(42), the combination coefficient and combination weight based on ICWGT can be calculated, as shown in Table 6.

Therefore, the combined weights and the weights calculated by EWM and FAHP can be obtained, as shown in Figures $8-10$.

In order to verify the rationality of the combined weighting of ICWGT, formula (43) was used to calculate the average degree of difference and was compared with CWGT [26], optimal planning [13], and product normalization method, as shown in Table 7.

As can be seen from Table 7, compared with other methods, the average difference of the second-level indicators of the three first-level indicators based on ICWGT is the smallest, so it can be concluded that ICWGT is reasonable for combined weighting and has superior performance.

\section{Results and Discussion}

4.1. Calculation of System Effectiveness. According to the normalized value of the sample data in Table 4 and combined weights in Table 6 , the value of first-level indicators of each sample can be obtained. The results are shown in Table 8. 


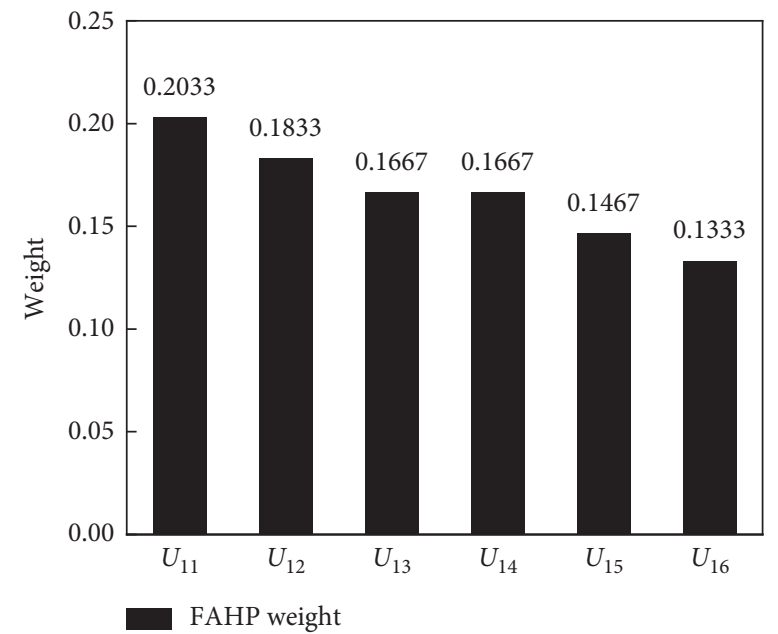

FIgURE 5: Weight of each indicator of combat capability based on FAHP.

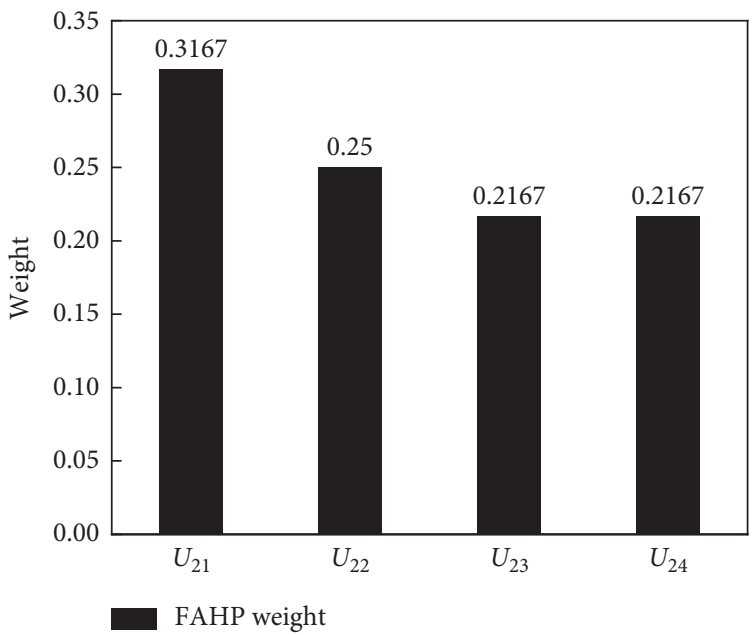

FIGURE 6: Weight of each indicator of adversarial ability based on FAHP.

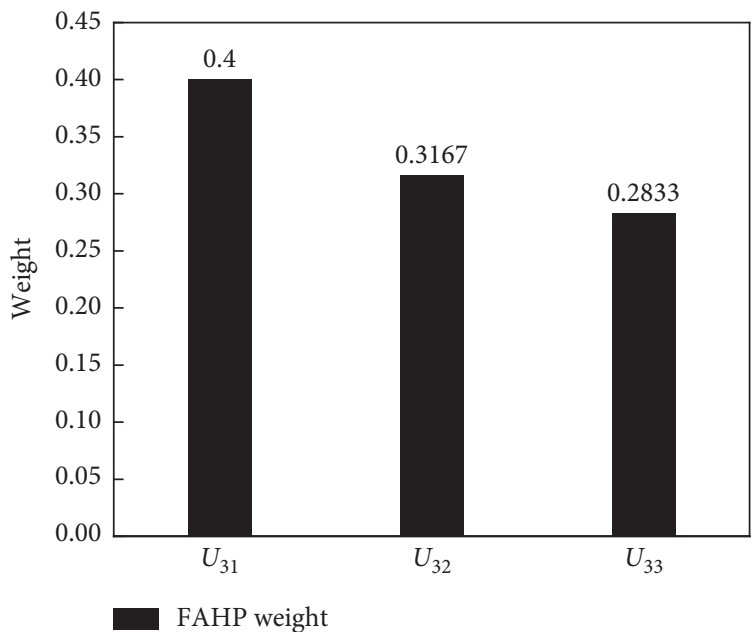

FIGURE 7: Weight of each indicator of state loss based on FAHP. 
TABLE 6: Combination coefficient and combination weight based on ICWGT.

\begin{tabular}{|c|c|c|c|c|}
\hline $\begin{array}{l}\text { First-level } \\
\text { indicators' code }\end{array}$ & Second-level indicators' code & $\begin{array}{c}\text { Combination coefficient } \\
\text { based on EWM }\end{array}$ & $\begin{array}{c}\text { Combination coefficient } \\
\text { based on FAHP }\end{array}$ & $\begin{array}{c}\text { Combination } \\
\text { weight }\end{array}$ \\
\hline \multirow{6}{*}{$U_{1}$} & $U_{11}$ & \multirow{6}{*}{0.5383} & \multirow{6}{*}{0.4617} & 0.0997 \\
\hline & $U_{12}$ & & & 0.2035 \\
\hline & $U_{13}$ & & & 0.2020 \\
\hline & $U_{14}$ & & & 0.1356 \\
\hline & $U_{15}$ & & & 0.1312 \\
\hline & $U_{16}$ & & & 0.2280 \\
\hline \multirow{4}{*}{$U_{2}$} & $U_{21}$ & \multirow{4}{*}{0.5357} & \multirow{4}{*}{0.4643} & 0.3763 \\
\hline & $U_{22}$ & & & 0.2923 \\
\hline & $U_{23}$ & & & 0.1165 \\
\hline & $U_{24}$ & & & 0.2150 \\
\hline \multirow{3}{*}{$U_{3}$} & $U_{31}$ & \multirow{3}{*}{0.5146} & \multirow{3}{*}{0.4854} & 0.4584 \\
\hline & $U_{32}$ & & & 0.2829 \\
\hline & $U_{33}$ & & & 0.2587 \\
\hline
\end{tabular}

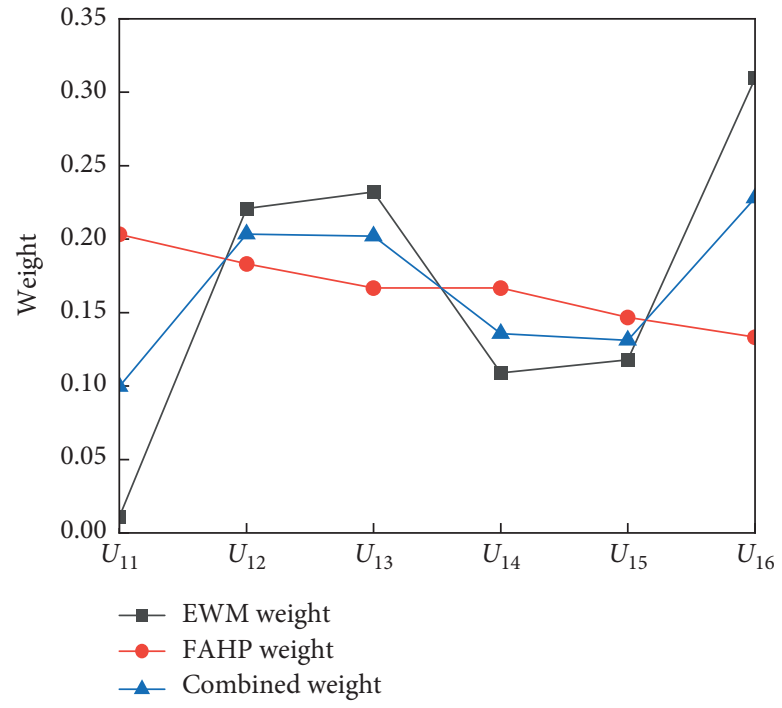

FIgURE 8: Weight of each indicator of combat capability.

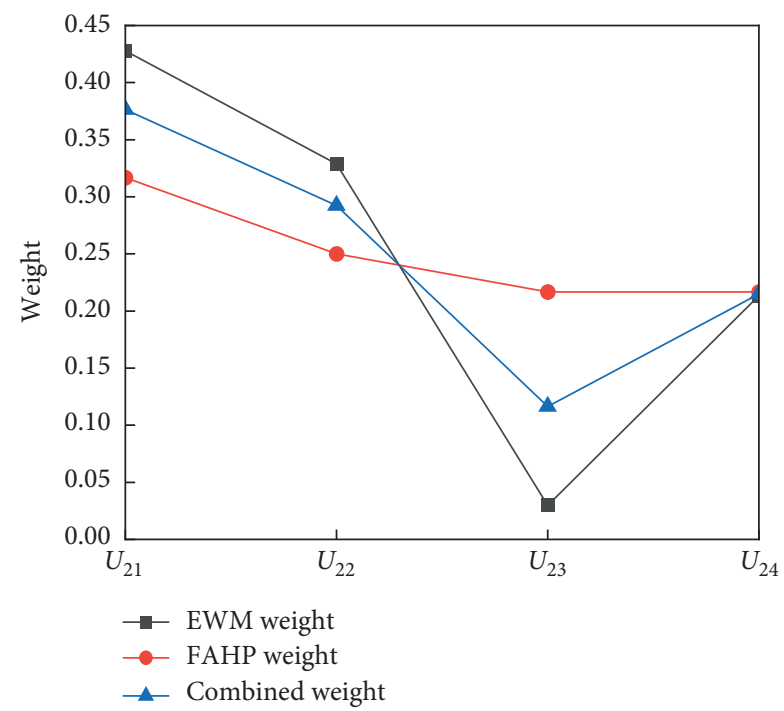

FIgURE 9: Weight of each indicator of adversarial ability. 


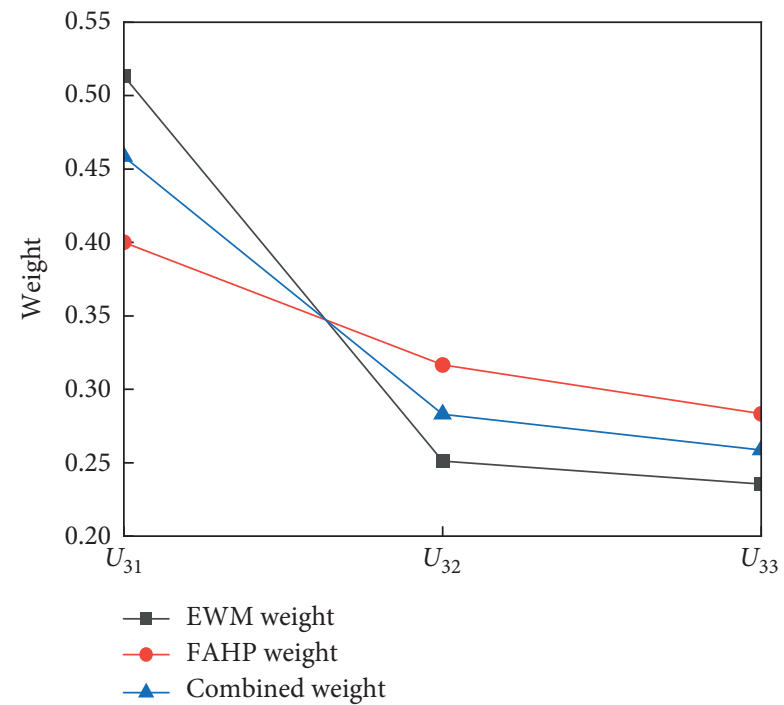

FIgURE 10: Weight of each indicator of state loss.

TABLE 7: Calculation of average difference based on different combination weighting methods.

\begin{tabular}{|c|c|c|c|c|}
\hline First-level indicators' code & CWGT [26] & Optimal planning [13] & Product normalization method & ICWGT \\
\hline$\overline{U_{1}}$ & 0.1899 & 0.4348 & 0.2332 & 0.1899 \\
\hline$U_{2}$ & 0.2530 & 0.1459 & 0.2389 & 0.1459 \\
\hline$U_{3}$ & 0.1910 & 0.0855 & 0.1824 & 0.0806 \\
\hline
\end{tabular}

TABLe 8: Value of first-level indicators of data samples.

\begin{tabular}{|c|c|c|c|c|c|c|c|}
\hline First-level indicators' code & $V_{11}$ & $V_{12}$ & $V_{13}$ & $V_{14}$ & $V_{15}$ & $V_{21}$ & $V_{22}$ \\
\hline$\overline{U_{1}}$ & 0.7332 & 0.6722 & 0.7432 & 0.7432 & 0.7432 & 0.4481 & 0.5126 \\
\hline$U_{2}$ & 0.7232 & 0.7232 & 0.7232 & 0.7232 & 0.7816 & 0.6248 & 0.7293 \\
\hline$U_{3}$ & 0.8342 & 0.8342 & 0.8342 & 0.8800 & 0.8342 & 0.3342 & 0.5083 \\
\hline
\end{tabular}

After obtaining the evaluation value of the first-level indicators, in order to obtain the inherent capability of the system, $U_{1}, U_{2}$, and $U_{3}$ need to be aggregated. Since the three indicators conform to strong mutual relations in the evaluation system and in order to simplify the model and prevent the influence of too many subjective factors on its effectiveness evaluation value, we use multiplication aggregation for the aggregation here. Therefore, we can calculate the inherent capability value $c$ of the system, as shown in Table 9.

Since MEMLS is still demonstrating research, there is no accurate data on MTBF, MTTR, and MLDT. In order to further study the effectiveness of the missile electromagnetic launch in the research stage, it is assumed that the reliability and maintainability indicators of the EMLS and the active launch system are the same, taking $\mathrm{MTBF}=1000 \mathrm{~h}$, $\operatorname{MTTR}=50 \mathrm{~h}, \quad \mathrm{MLDT}=20 \mathrm{~h}, \quad$ and $t=1 \mathrm{~h} . \quad a_{1} \cdot \mathrm{d}_{11}=$ 0.9336 can be calculated. The system effectiveness evaluation value can be obtained, as shown in Table 10.

In the table, $V_{11}$ to $V_{15}$ represent MEMLS with different battle targets, and $V_{21}$ to $V_{22}$ represent missile launching systems using thermal launching and cold launching technologies, respectively.
TABLE 9: Inherent capabilities of different data samples.

\begin{tabular}{cccccccc}
\hline & $V_{11}$ & $V_{12}$ & $V_{13}$ & $V_{14}$ & $V_{15}$ & $V_{21}$ & $V_{22}$ \\
\hline$C$ & 0.7619 & 0.7402 & 0.7654 & 0.7791 & 0.7855 & 0.4540 & 0.5749 \\
\hline
\end{tabular}

TABLe 10: System effectiveness evaluation values.

\begin{tabular}{cccccccc}
\hline & $V_{11}$ & $V_{12}$ & $V_{13}$ & $V_{14}$ & $V_{15}$ & $V_{21}$ & $V_{22}$ \\
\hline$E$ & 0.7113 & 0.6911 & 0.7146 & 0.7274 & 0.7334 & 0.4239 & 0.5368 \\
\hline
\end{tabular}

4.2. Discussion of Calculation Results. Combining the indicators of MEMLS's demonstration program, this paper makes a further analysis of the results of the effectiveness evaluation and mainly obtains the following two points.

(1) According to Table 10, the effectiveness of EMLS with different battle targets is higher than that of launch systems using other technologies. This proves that from the perspective of effectiveness, it is necessary to change the traditional launching method and study the missile electromagnetic launching technology.

(2) Combining Tables 3 and 10, comparing $V_{11}, V_{12}$ and $V_{13}, V_{14}$, it can be seen that shortening the 
acceleration time and reducing the ablation degree of the launcher can significantly improve the effectiveness of the EMLS, which is also important for the missile electromagnetic launch technology. Comparing $V_{11}$ and $V_{13}$, it can be seen that the effectiveness has not been greatly improved when the initial ejection velocity is increased. This is because the original launch system can already meet the initial ejection velocity requirements of the missile. If the focus of the EMLS is on the improvement of the initial ejection velocity, there is no greater value and significance. Comparing $V_{13}$ and $V_{15}$, it can be seen that the effect of electromagnetic anti-interference ability on the system effectiveness is also obvious. This is because the EMLS adds a lot of electromagnetic components, and it is also easy to be interfered by the enemy's electromagnetic interference machine. Therefore, this is also a research focus of MEMLS.

\section{Requirements of Improving System Effectiveness on Reliability and Maintainability}

Section 4.2 discusses the effect of some indicators of inherent capability $\mathbf{C}$ on the effectiveness based on the results of the effectiveness calculation. In order to further improve the effectiveness of the system, according to formula (1), the effectiveness of the available system is also related to availability $\mathbf{A}$ and dependability $\mathbf{D}$. Availability $\mathbf{A}$ and dependability $\mathbf{D}$ are determined by four indicators, namely, reliability indicator MTBF, maintainability indicator MTTR, MLDT, and total task duration $t$. The total duration of the missile's execution of the combat mission is generally a fixed value, and it is assumed here that $t=1 \mathrm{~h}$. MLDT is related to the comprehensive support strength of a country's military, and it is assumed here that MLDT $=20 \mathrm{~h}$. In order to analyze how to further improve system effectiveness, the relationship among MTBF, MTTR, and effectiveness is specifically analyzed. According to the calculation results above, take inherent capability value $c=0.7855$ here; from formulas (44) and (45), the relationship model among system effectiveness and reliability and maintainability indicators can be obtained as

$$
\begin{aligned}
\mathrm{MTBF} & =\frac{E(\mathrm{MTTR}+\mathrm{MLDT})}{c \cdot e^{-(t / \mathrm{MTBF})}-E}, \\
\mathrm{MTTR} & =\frac{c \cdot \mathrm{MTBF} \cdot e^{-(t / \mathrm{MTBF})}}{E}-\mathrm{MTBF}-\mathrm{MLDT} .
\end{aligned}
$$

In the following, the effects of improving system efficiency on reliability and maintainability indicators are analyzed separately.

5.1. Impact of Improving System Effectiveness on Reliability Indicator MTBF. Perform difference analysis and differential analysis on the indicators. First conduct a difference analysis. In formula (53), assuming that MTTR is unchanged, when $E$ changes by $\Delta E$, the amount of change in MTBF can be obtained as

$$
\Delta \mathrm{MTBF}=\frac{(\mathrm{MTTR}+\mathrm{MLDT}) \cdot c \cdot e^{-(t / \mathrm{MTBF})}}{\left[c \cdot e^{-(t / \mathrm{MTBF})}-(E+\Delta E)\right]\left(c \cdot e^{-(t / \mathrm{MTBF})}-E\right)} \cdot \Delta E .
$$

According to formula (55), when the system performance increases, $\Delta E>0$, then $\Delta \mathrm{MTBF}>0$, so MTBF increases with the increase of $E$.

The following is a differential analysis. In formula (53), assuming that the MTTR is unchanged, when $E$ changes by $\Delta E$, it can be obtained by using the implicit function derivation.

$$
\begin{aligned}
\mathrm{MTBF}^{\prime} & =\frac{\mathrm{MTBF}+\mathrm{MTTR}+\mathrm{MLDT}}{c \cdot e^{-(t / \mathrm{MTBF})}(1+(t / \mathrm{MTBF})-E}, \\
\Delta \mathrm{MTBF} & =\frac{\mathrm{MTBF}+\mathrm{MTTR}+\mathrm{MLDT}}{c \cdot e^{-(t / \mathrm{MTBF})}(1+(t / \mathrm{MTBF}))-E} \cdot \Delta E .
\end{aligned}
$$

It can also be obtained from formula (57) that when the system effectiveness increases, $\Delta E>0$, then $\Delta \mathrm{MTBF}>0$, so the MTBF increases with the increase of $E$. On the basis of differential analysis, taking MTTR $=50 \mathrm{~h}$ as an example and taking different values of $E$, after calculation, Table 11 can be obtained.

It can be seen from Table 11 that as the value of $E$ becomes larger and larger, the rate of change of MTBF becomes faster and faster, and the limit value is infinite. That is to say, in order to continue to improve the effectiveness of the system, the reliability indicator must be further improved, and it also means that the cost of the system will also increase significantly.

According to the above assumptions, taking MTTR in order of $50,45,40,35,30,25$, and 20 , the curve can be obtained as shown in Figure 11.

As can be seen from Figure 11, the reliability indicator MTBF increases with the effectiveness $E$. This is consistent with the conclusion of the difference analysis above, and its change trend is also consistent with Table 11.

5.2. Impact of Improving System Effectiveness on Maintainability Indicator MTTR. Similarly, perform difference analysis and differential analysis on maintainability indicator. First, conduct a difference analysis. In formula (54), assuming that MTBF is unchanged, when $E$ changes by $\Delta E$, the amount of change in MTTR can be obtained as

$$
\Delta \operatorname{MTTR}=-\frac{c \cdot \mathrm{MTBF} \cdot e^{-(t / M T B F)}}{(E+\Delta E) E} \cdot \Delta E .
$$

According to formula (55), when the system effectiveness increases, $\Delta E>0$, then $\Delta \mathrm{MTTR}<0$, so MTTR decreases with the increase of $E$.

The following is a differential analysis. In formula (54), assuming that the MTBF is unchanged, when $E$ changes by $\Delta E$, it can be obtained by using derivation. 
TABLE 11: MTBF changes with $E$.

\begin{tabular}{lccccccccc}
\hline$E$ & 0.7 & 0.71 & 0.72 & 0.73 & 0.74 & 0.75 & 0.76 & 0.77 & 0.7855 \\
\hline MTBF $^{\prime}$ & 7629 & 9784 & 12999 & 18106 & 26939 & 44254 & 85768 & 232135 & $\infty$ \\
MTBF & 582 & 669 & 781 & 935 & 1156 & 1501 & 2117 & 3528 & $\infty$ \\
\hline
\end{tabular}

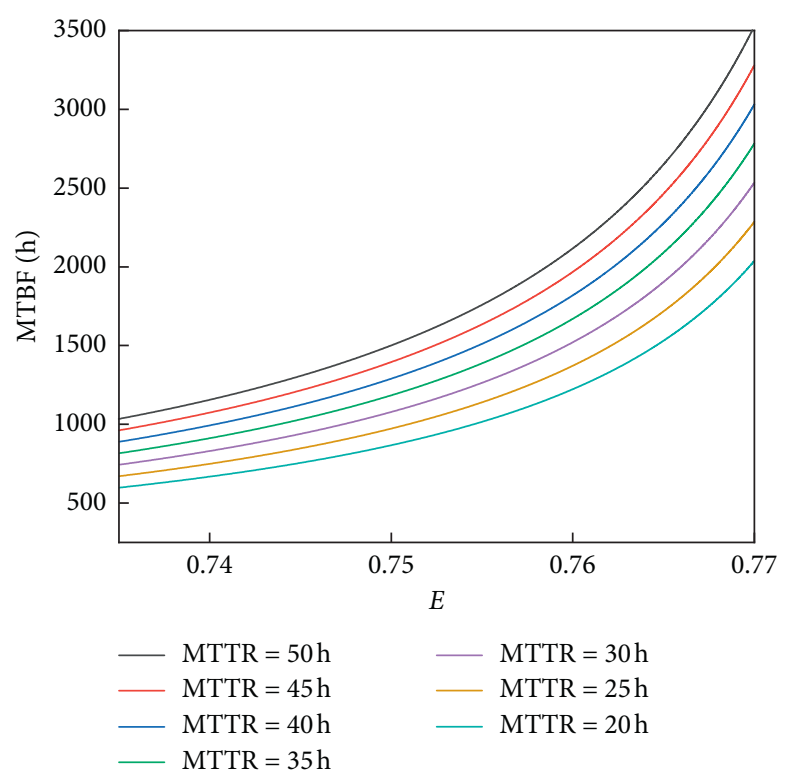

Figure 11: Relationship between system effectiveness and reliability indicator MTBF.

TABLE 12: MTTR changes with $E$.

\begin{tabular}{lccccccccc}
\hline$E$ & 0 & 0.1 & 0.2 & 0.3 & 0.4 & 0.5 & 0.6 & 0.7 & 0.7855 \\
\hline MTTR' $^{\prime}$ & $-\infty$ & -78471 & -19618 & -8719 & -4905 & -3139 & -2280 & -1602 & -1272 \\
MTTR & $\infty$ & 6827 & 2904 & 1596 & 942 & 549 & 288 & 101 & -21 \\
\hline
\end{tabular}

$$
\begin{aligned}
\operatorname{MTTR}^{\prime} & =-\frac{c \cdot \mathrm{MTBF} \cdot e^{-(t / \mathrm{MTBF})}}{E^{2}}, \\
\Delta \mathrm{MTTR} & =-\frac{c \cdot \mathrm{MTBF} \cdot e^{-(t / \mathrm{MTBF})}}{E^{2}} \cdot \Delta E .
\end{aligned}
$$

It can also be obtained from formula (60) that when the system effectiveness increases, $\Delta E>0$, then $\Delta \mathrm{MTTR}<0$, so MTTR decreases as $E$ increases. On the basis of differential analysis, taking $\mathrm{MTBF}=1000 \mathrm{~h}$ as an example and taking different values of $E$, after calculation, Table 12 can be obtained.

It can be found that $E$ changes very rapidly before taking 0.4 , and after $E>0.6$, the speed becomes slow. This shows that in order to further significantly improve the system effectiveness, it is only necessary to reduce the MTTR by dozens, but at the same time, since the normal range of the MTTR value itself is small, each point of improvement requires a larger cost.

According to the above assumptions, taking MTBF as $1000,1500,2000,2500,3000,3500$, and 4000 in turn, the curve can be obtained as shown in Figure 12.

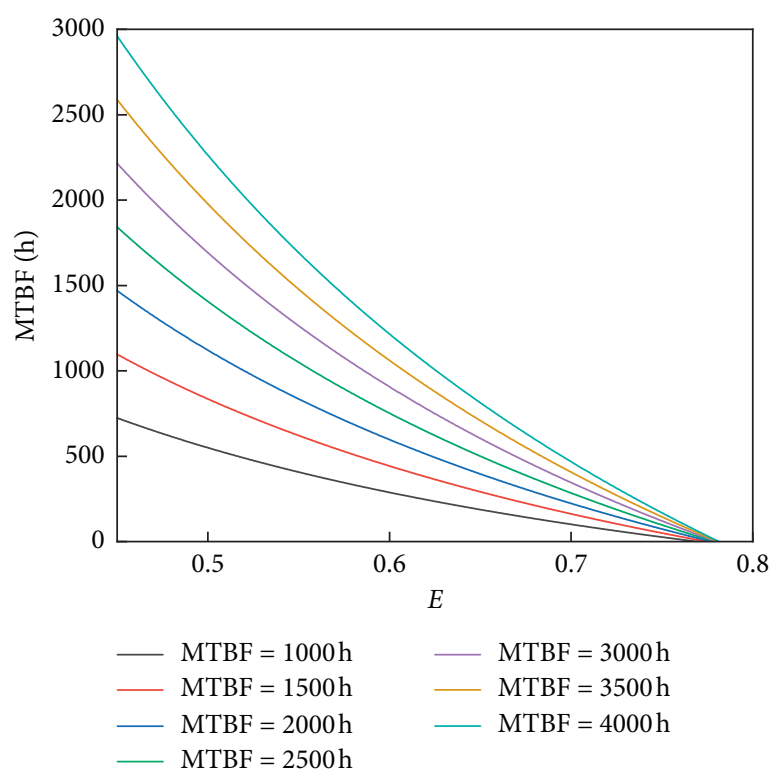

Figure 12: Relationship between system effectiveness and maintainability indicator MTTR. 


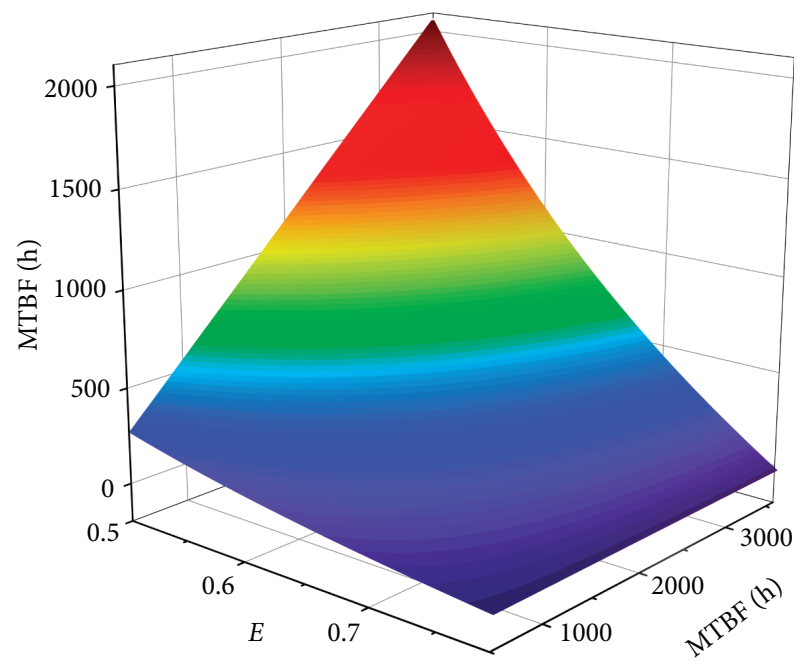

Figure 13: Relationship among system effectiveness and two indicators.

As can be seen from Figure 12, the maintainability indicator MTTR decreases as the effectiveness $E$ increases. This is consistent with the conclusion of the above difference analysis, and its change trend is also consistent with Table 12.

5.3. Comprehensive Analysis of Indicators. After analyzing the relationship among system effectiveness, MTBF, and MTTR, a comprehensive analysis of indicators is conducted. From formula (54), when $E$ is $0.5 \sim 0.7855$, Figure 13 can be obtained.

It can be seen from Figure 13 that when the system effectiveness $E$ increases, the MTBF increases and the MTTR decreases. Since MTBF is related to the reliability of the equipment itself, use process, environment, and other factors and MTTR is related to the detection and maintenance capabilities of the department, there is no direct relationship between the two indicators. According to the system effectiveness requirements, the value of the indicators can be determined to achieve the goal of improving effectiveness.

\section{Conclusion}

The main innovations and contributions of this paper are as follows:

(1) It is the first time to publicly evaluate the entire system of MEMLS to provide basis and guidance for the design and improvement of the system.

(2) The paper is based on the ADC model and uses EWM-FAHP-ICWGT to calculate the weights of the indicators. The calculation process is reasonable and innovative, and the calculation results are superior.

(3) The method proposed in the paper is applicable to any quantifiable indicator model and has good scalability.

The main conclusions of this paper are as follows:
(1) It can be obtained from Section 4 that the effectiveness of EMLS using different battle targets is higher than that of launch systems using other technologies. This proves the necessity of changing the traditional launching method and studying missile electromagnetic launching technology from the perspective of efficiency. At the same time, it also proves the effectiveness of the model in this paper.

(2) It can be obtained from Section 4 that the effectiveness of the MEMLS can be improved by shortening the acceleration time and reducing the ablation degree of the launcher. It is necessary to study the problem of high pulse current discharge, the dynamic analysis of the armature, the integrated projectile, and the ablative material and infrared stealth material. And increasing initial ejection velocity has little effect on effectiveness. At the same time, the research on the system's electromagnetic anti-interference ability is also a key point.

(3) It can be obtained from Section 5 that system effectiveness can be improved by changing the values of reliability and maintainability indicators. Through difference analysis and differential analysis, it can be concluded that the change of reliability indicator MTBF is gradually accelerated with the change of effectiveness and has a positive relationship with effectiveness. The change of maintainability indicator MTTR gradually slows down with the change of effectiveness and has a negative relationship with effectiveness. In order to further improve the effectiveness, it is necessary to comprehensively increase MTBF and reduce MTTR in order to achieve combat objectives. This can be achieved by increasing the frequency of equipment routine maintenance and a fast and effective spare parts repair and replacement system. At the same time, it also requires the strengthening of mutual communication between the entire military industries.

The shortcomings of this paper as well as the next research focus and suggestions are as follows:

(1) The disadvantage of the paper is that the research on the reliability and maintainability of the system is relatively simple, only from the mathematical model; at the same time, the weight calculation process is relatively complicated, which is not conducive to the direct use of the program demonstration personnel.

(2) In order to design the optimal MELS, the next step is to further study the reliability and maintainability indicators from the perspective of system design; at the same time, the system scheme indicators and the resulting effectiveness values should be fitted so that the effectiveness values can be quickly obtained from the demonstration scheme indicators. Due to the variability of the short working process of MEMLS, it is also the next step to consider the dynamics of indicators and weights. 


\section{Data Availability}

The data used to support the findings of this study are available from the corresponding author upon request.

\section{Conflicts of Interest}

The authors declare that there are no conflicts of interest regarding the publication of this paper.

\section{Acknowledgments}

This research was funded by the National Natural Science Foundation of China (71601180).

\section{References}

[1] S.-K. Lu, D.-X. Hua, Y. Li, F.-Y. Cui, and P.-Y. Li, "Stiffness calculation method and stiffness characteristic analysis of bolted connectors," Mathematical Problems in Engineering, vol. 2019, Article ID 6206092, 11 pages, 2019.

[2] C. Deng, C. Ye, J. Yang, S. Sun, and D. Yu, "A novel permanent magnet linear motor for the application of electromagnetic launch system," IEEE Transactions on Applied Superconductivity, vol. 30, no. 4, pp. 1-5, 2020.

[3] S. Ma, X. Yu, and Z. Li, "A review of the current research situation of inductive pulsed-power supplies for electromagnetic launch," Transactions of China Electrotechnical Society, vol. 30, no. 24, pp. 222-228, 2015.

[4] C. Gong, X. Yu, and X. Liu, "Continuous emission scheme and its simulation for capacitor-based railgun system," Transactions of China Electrotechnical Society, vol. 28, no. S2, pp. 111-115+121, 2013.

[5] Y. Wu, J. Lu, X. Long, R. Zhou, and Y. Liu, "Optimization of energy transfer rate of hybrid energy storage system in electromagnetic launch," High Voltage Engineering, vol. 45, pp. 3715-3720, 2019.

[6] S. Xiong, J. Lu, Y. Zheng, and D. Zeng, "Research on discharge performance evaluation system of storage pulse power supply," Journal of Naval University of Engineering, vol. 31, 2019.

[7] L. Xu, G. Chen, and Q. Li, "Ultra-local model-free predictive current control based on nonlinear disturbance compensation for permanent magnet synchronous motor," IEEE Access, vol. 8, pp. 127690-127699, 2020.

[8] R. Shao, Z. Fang, S. Gao et al., "G-BDP-ADC model for effectiveness evaluation of low orbit satellite communication system in the context of poor information," IEEE Access, vol. 7, pp. 157489-157505, 2019.

[9] H. Pirzadeh and A. L. Swindlehurst, "Spectral efficiency of mixed-ADC massive MIMO," IEEE Transactions on Signal Processing, vol. 66, no. 13, pp. 3599-3613, 2018.

[10] C. Gui, "Evaluation of unmanned equipment operational effectiveness based on ADC model," in Proceedings of the 2018 10th International Conference on Intelligent Human-Machine Systems and Cybernetics (IHMSC), pp. 299-303, Hangzhou, China, August 2018.

[11] G.-C. Niu, Y. Wang, Z. Hu, Q. Zhao, and D.-m. Hu, “Application of AHP and EIE in reliability analysis of complex production lines systems," Mathematical Problems in Engineering, vol. 2019, Article ID 7238785, 10 pages, 2019.

[12] Z. Gao, M. Li, F. Gao, and X. Wang, "Fuzzy comprehensive evaluation on body parts' weight coefficients towards sitting comfort based on AHP to limit entropy method,"
Mathematical Problems in Engineering, vol. 2019, Article ID 3826468, 11 pages, 2019.

[13] X. L. Zhai, Z. Z. Lin, F. S. Wen, and J. S. Huang, "Power quality comprehensive evaluation based on combination weighting method," in Proceedings of the International Conference on Sustainable Power Generation \& Supply (SUPERGEN 2012), September 2013.

[14] C. Stevens, "Weapon system effectiveness industry advisory committee," SAE Technical Paper Series, 1964.

[15] L. Mkrtchyan, L. Podofillini, and V. N. Dang, "Bayesian belief networks for human reliability analysis: a review of applications and gaps," Reliability Engineering \& System Safety, vol. 139, pp. 1-16, 2015.

[16] P. Baraldi, L. Podofillini, L. Mkrtchyan, E. Zio, and V. N. Dang, "Comparing the treatment of uncertainty in bayesian networks and fuzzy expert systems used for A human reliability analysis application," Reliability Engineering \& System Safety, vol. 138, pp. 176-193, 2015.

[17] M. T. Davis, M. J. Robbins, and B. J. Lunday, “Approximate dynamic programming for missile defense interceptor fire control," European Journal of Operational Research, vol. 259, no. 3, pp. 873-886, 2017.

[18] I. S. Golubev and V. G. Svetiov, Air Defense Missile Design, Moscow Aviation University Press, Moscow, Russia, 2001.

[19] C. Li, J. Qin, J. Li, and Q. Hou, "The accident early warning system for iron and steel enterprises based on combination weighting and grey prediction model GM (1, 1)," Safety Science, vol. 89, pp. 19-27, 2016.

[20] A. Eiger, P. B. Mirchandani, and H. Soroush, "Path preferences and optimal paths in probabilistic networks," Transportation Science, vol. 19, no. 1, pp. 75-84, 1985.

[21] L. A. Zadeh, "Fuzzy sets as a basis for a theory of possibility," Fuzzy Sets and Systems, vol. 100, no. 1, pp. 9-34, 1999.

[22] N. Mou, C. Yang, and L. Zhang, "Evaluation of development potential of ports in the Yangtze river delta using FAHPentropy model," Sustainability, vol. 12, no. 2, p. 493, 2020.

[23] N. Chen, S. Wang, D. Fu, and J. Zhao, "Basic experimental evaluation on safety behavior competence of construction workers based on FAHP," Journal of Civil Engineering and Management, vol. 1, 2019.

[24] R. Fazel, J. Mehdi, M. Hossein, M. Honarbakhsh, and P. Farhad, "Occupational stress among nurses and pre-hospital emergency staff: application of fuzzy analytic hierarchy process (FAHP) method," EXCLI Journal, vol. 17, pp. 808824, 2018.

[25] X. Jiang, H. Wang, X. Guo, and X. Gong, "Using the FAHP, ISM, and MICMAC approaches to study the sustainability influencing factors of the last mile delivery of rural E-commerce logistics," Sustainability, vol. 11, no. 14, 2019.

[26] J. Cai, T. Wang, X. Xia, Y. Chen, H. Lv, and N. Li, "Analysis on the choice of livelihood strategy for peasant households renting out farmland: evidence from western poverty-stricken areas in China," Sustainability, vol. 11, no. 5, pp. 1-13, 2019. 